\title{
Combined Wastewater Treatment from Manganese Phosphate Coating: Coagulation-Ozonation-Fenton Reaction-Sorption
}

\author{
Jakub Duszczyk*, Adriana Zaleska-Medynska \\ Department of Environmental Technology, Faculty of Chemistry, University of Gdansk, \\ Str. Wita Stwosza 63, 80-308 Gdansk, Poland
}

Received: 2 October 2020

Accepted: 19 November 2020

\begin{abstract}
Wastewater arising from manganese phosphate coating contains plenty of biogenic compounds, heavy metals such as nickel, iron, manganese, and therefore also constitutes a serious threat to the environment. The combined method of removing pollutants from wastewater from manganese phosphate coating consisting of coagulation, ozonation and Fenton reaction allowed to reduce biogenic pollutants and remove heavy metals. Coagulation was performed with iron(III) chloride and Flopam AN 934 SHU flocculant. The Fenton reaction was performed in two combinations with and without the addition of iron(III) salt. Ozonation was carried out in a column with an ozone generator, and wastewater was treated in the following sequence: coagulation-ozonation-Fenton reaction. The results of experiments demonstrated that the use of combined methods of coagulation, ozonation and Fenton reaction have brought the expected outturns. As a result of using the Fenton reaction, the content of nitrates(V) was lowered from $13.0 \mathrm{mg} / 1$ to $0.012 \mathrm{mg} / 1$. Physico-chemical analyses of wastewater were performed using UV-VIS molecular spectrophotometry.
\end{abstract}

Keywords: industrial wastewater, wastewater treatment, phosphating wastewater treatment, fenton reaction, sewage ozonation, coagulation processc, manganese phosphate coating

\section{Introduction}

The industry is a manufacturer of one of the most toxic wastewaters in the economy. The fast-growing metallurgical economy produces two million tons of wastewater on a daily basis [1]. The main components most often wastewater from phosphate process include heavy metals such as $\mathrm{Cd}, \mathrm{Cr}, \mathrm{Cu}, \mathrm{Ni}, \mathrm{Pb}, \mathrm{Zn}$ [2] and

*e-mail: jakubduszczyk@onet.com.pl substances such as detergents or oils [2, 3]. Most of the produced extraneous substances from galvanic production often have teratogenic, mutagenic or carcinogenic properties in relation to the human body [4-6]. It is therefore very important that toxic and harmful substances from wastewater are removed before discharging them into sewage systems or directly into the environment.

The methods of industrial wastewater treatment include precipitation techniques with the use of calcium compounds $[7,8]$, coagulation methods with the use of aluminum and iron compounds [9, 10], treatment with 
the use of membrane methods [11], adsorption processes using zeolites or biomass [12], processes using nanofiltration or reverse osmosis [13, 14], electrodialysis [15-17], photocatalytic methods [18], ultrafiltration [19], purification with the use of activated carbon [20-22] as well as techniques of advanced oxidation with the use of ozone, hydrogen peroxide, UV radiation and Fenton's reagents $[23,24]$. Beside chemical methods, we should also list physical methods in wastewater treatment, such as sedimentation [25] or processes related to the equalization of wastewater flow, which mainly consist of buffering by introducing equalizing devices or such retaining the flowing wastewater [26]. Physical treatment of wastewater involves methods consisting in the separation of floating solid particles, which could prejudice further treatment [27-32].

One important branch is the automotive industry, which involves the production of car and truck construction elements as well as the manufacture of material handling equipment such as elevator components, conveyor belts, and cranes. These devices operate under extreme conditions, often in a humid environment, and therefore require protection against corrosion. One of the methods for protecting such elements against corrosion is the manganese phosphate coating process, which is the source of wastewater containing orthophosphates, nitrates(V), manganese, nickel, chlorides, sulphates(VI), sodium cations; potassium and even titanium and zirconium compounds, as well as nitrogen compounds such as nitro compounds, including nitroguanidine [3334]. Due to the high content of these compounds and their chemical diversity, such sewage is particularly burdensome and difficult to treat.

In this regard, the aim of this paper is to develop a method that would allow for pretreatment of wastewater generated in the manganese phosphate coating process. Real wastewater samples have been characterized and treated using such processes as coagulation, sorption with the use of activated carbon and zeolite, ozonation and the Fenton reaction. Sewage treatment efficiency was assessed on the basis of 16 parameters determined in the sewage before and after treatment. It is the first such paper to have discussed the application of ozonation and the Fenton method for wastewater treatment from manganese phosphate coating. To this day, these methods have mainly been used for treatment of sewage containing organic compounds, including dyes [35-40]. Due to the content of iron and manganese phosphate compounds in the sewage, the use of oxidation methods allowed to reduce the total amount of these substances, which can be easily separated from the sewage with a filter press.

\section{Material and Methods}

In this paper, we used wastewater from manganese phosphate coating from Mayr Polska based at
Hetmańska 1 Street, 63-500 Ostrzeszów. Also used was raw sewage collected directly from the washings buffer tank, in which acidic sewage is mixed after digestion in hydrochloric acid and alkaline acid after degreasing in a solution of potassium and sodium hydroxide.

\section{Preparation of Sewage Samples}

The wastewater sample treated with coagulation and the raw wastewater sample were collected during $8.0 \mathrm{~h}$ in the total amount of $2.5 \mathrm{dm}^{3}$. The samples prepared in this way were poured and an average sample was obtained. Wastewater samples were collected in accordance with the Polish Standard for wastewater sampling [41]. Next, they were subjected to a physicochemical analysis, carried out in accordance with the methodology of Polish and European Standards, and further treatment in the case of raw sewage.

Parameters such as $\mathrm{pH}$, electrical conductivity as well as temperature were determined using multifunction meter CX-601 (ELMETRON, Poland). Turbidity was measured using a 2100Q turbidity meter (HACH, USA). The content of biogenic compounds: nitrate nitrogen (III) $\mathrm{N}-\mathrm{NO}_{2}$, nitrate(V) $\mathrm{NO}_{3}$, ammonium nitrogen $\mathrm{N}_{-} \mathrm{NH}_{4}$ and orthophosphates( $(\mathrm{V}) \quad \mathrm{PO}_{4}$ was determined by colorimetric method using the MachereyNagel cuvette tests by means of a PF-12 photometer (Macherey-Nagel, Germany). Metals such as Fe, Mn, $\mathrm{Cr}$ were established utilizing the spectrophotometric method with a UV-VIS spectrophotometer (METASH, China). Chlorides, hardness, alkalinity were calculated by means of titration methods. All devices were calibrated before use as stated by the manufacturer's instructions.

\section{Coagulation}

Wastewater from manganese phosphate coating with the composition presented in Table 2 was subjected to neutralization. Coagulation, neutralization with limewash and flocculation were carried out in an automatic system under industrial conditions in three separate reaction tanks with mechanical mixers, each with a capacity of $0.369 \mathrm{~m}^{3}$. The process took place next to the manganese phosphate coating process in Mayr (Mayr Polska, Hetmańska 1 str., 63-500 Ostrzeszów, Poland). Iron(III) chloride solution with a concentration of $40 \% \quad(\mathrm{~m} / \mathrm{m})$ stabilized with hydrochloric acid was used as a coagulant. A calcium hydroxide suspension prepared in a $1.2 \mathrm{~m}^{3}$ tank was used as a neutralizing agent. A suspension of $4 \%$ concentration was used in a dose determined depending on the sewage feed, until a $\mathrm{pH}$ of 10.1 was obtained in the neutralization chamber. FLOPAM AN 934 SHU in the form of a solution with a concentration of $80 \mathrm{~g} / \mathrm{m}^{3}$ was used as a flocculant.

The diagram of the manganese phosphate coating wastewater neutralizer used by Mayr Polska is shown in Fig. 1. 


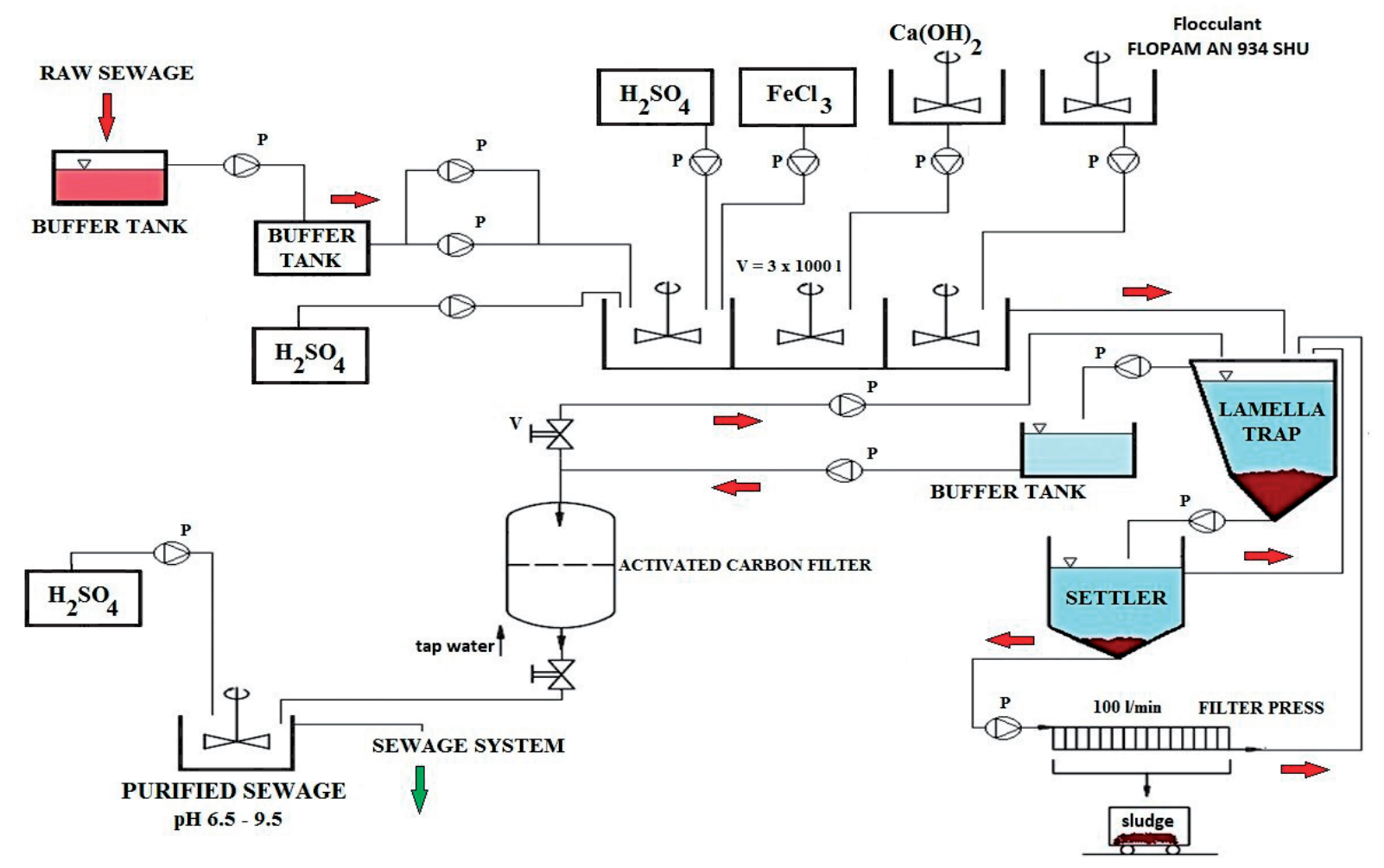

Fig. 1. Manganese phosphate coating sewage treatment plant.

\section{Ozonation}

Ozonation was performed in the barbotage ozone column at the Department of Environmental Technology, Faculty of Chemistry, University of Gdańsk (Gdańsk, Poland). The process was carried out with the use of $3 \mathrm{dm}^{3}$ of raw sewage and $3 \mathrm{dm}^{3}$ of treated sewage during 1 hour. The unreacted ozone was collected in a washer containing $150 \mathrm{ml}$ of $5 \%(\mathrm{w} / \mathrm{m})$ $\mathrm{KI}$ solution and analyzed. During ozonation in $21^{\circ} \mathrm{C}$, samples for physico-chemical analysis were taken at 10-minute intervals.

\section{Fenton Reaction}

For this purpose, a solution of $\mathrm{FeCl}_{3}$ with a concentration of $30 \%(\mathrm{~m} / \mathrm{m})$ in the amount of $0.1 \mathrm{ml}$ and $35 \%(\mathrm{~m} / \mathrm{m})$ solution of $\mathrm{H}_{2} \mathrm{O}_{2}$ in varying amounts from 1 to $3 \mathrm{ml}$ were added to a sample of $500 \mathrm{ml}$. Also, sulfuric acid (VI) $95 \%(\mathrm{~m} / \mathrm{m})$ in the amount of $0.05 \mathrm{ml}$ was introduced into the measuring system. The reaction was carried out for 30 minutes in $21^{\circ} \mathrm{C}$. The test samples were taken at regular intervals.

\section{Cleaning on Mixed Bed of Activated Charcoal-Zeolite}

Wastewater sorption was carried out in the activated carbon-zeolite structure in a dual-column sorption system. The process schema is shown in Fig. 2; it involved the use of activated coconut charcoal with granulation of $0.6-2.36 \mathrm{~mm}(30-8 \mathrm{mesh})$ (SigmaAldrich) and granulated zeolite (Sigma-Aldrich). In order to purify the wastewater, a sewage sample of variable volume was passed through a column packed with activated carbon and zeolite in the amount of $100.0 \mathrm{~g}$ each.

\section{Results and Discussion}

The study involved the use of raw wastewater from manganese phosphate coating. Wastewater samples were prepared in accordance with the methodologies of Polish and European standards as listed in Tables 2, 3.

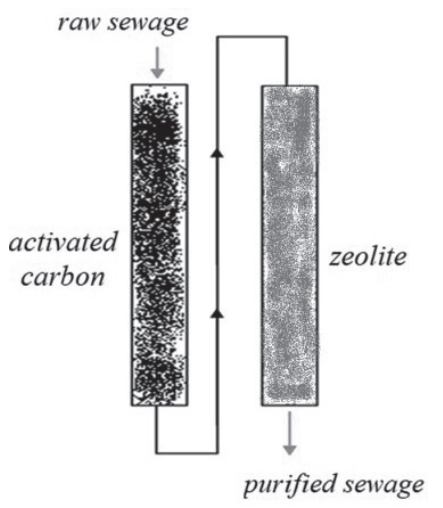

Fig. 2. Wastewater treatment system in the activated carbonzeolite structure. 
The study was conducted to compare the efficiency of electroplating wastewater treatment from manganese phosphate coating by coagulation using iron(III) chloride as a coagulant and waste sulfuric(VI) acid, calcium hydroxide as a precipitant and flocculation with the use of FLOPAM AN 934 SHU flocculant solution with a concentration of $80 \mathrm{~g} / \mathrm{m}^{3}$. These methods were compared with ozonation and purification with the Fenton reaction. The method of cleaning with sorption was also used.

\section{Characteristics of Wastewater from Manganese Phosphate Coating}

Characterization of wastewater from unit processes, unmixed with one another, was prepared on the basis of 120 samples collected between January and June of 2020, and shown in Table 1.

Wastewater from manganese phosphate coating is characterized by a variable composition (Table 1). It is most often determined by the daily or weekly production, which is closely related to the use of water for rinsing of the obtained coatings. Manganese phosphate coating involves the production of several types of wastewater, which are most often collected in the same tank where all washings from the technological process are kept. This type of sewage includes diluted sewage, which is represented by acid-alkaline washings
(Table 1). This wastewater is produced in the amount of about 2.5-3.0 $\mathrm{m}^{3}$ within eight hours. Such washings are formed at the stage of rinsing of galvanized elements such as:

- after the step of washing in strongly alkaline baths. Washing bath $\mathrm{pH}>11.0$, sodium and potassium hydroxide concentration converted to $\mathrm{KOH}: 0.5 \mathrm{~mol}$, temp. $>80^{\circ} \mathrm{C}$. These washings are produced during rinsing and degreasing of metal elements undergoing further treatment. Aqueous washing liquids contain the remains of $\mathrm{NaOH}, \mathrm{KOH}$, phosphates, surfactants, detergents and emulsifying agent,

- after digestion in hydrochloric acid. These washings contain chromium $\mathrm{Cr}^{6+}$, nickel $\mathrm{Ni}^{2+}$, large amounts of iron $\mathrm{Fe}^{2+}$ from the etched metal,

- after galvanization in a phosphating bath (bath composition: $\mathrm{H}_{3} \mathrm{PO}_{4}, \mathrm{Mn}^{2+}$ compounds, $\mathrm{Ni}^{2+}$ compounds, nitrates(V), sometimes chlorates and nitro compounds) [33-34].

Another type of sewage is concentrated wastewater from surface activation processes just prior to phosphating; surface passivation as well as wastewater from the hydrochloric acid vapor scrubber.

This type of sewage includes wastewater from the periodic replacement of the process baths with new ones, e.g. wastewater from activation of the metal surface. Depending on the type of activating agent, it contains large amounts of inorganic acids or

Table 1. Typical composition of washings from unit manganese phosphate coating processes. Data averaged on the basis of 6-month studies, i.e. 120 trials.

\begin{tabular}{|c|c|c|c|c|c|}
\hline \multirow[b]{2}{*}{ Parameter } & \multirow[b]{2}{*}{ Unit } & \multicolumn{4}{|c|}{ Type of absorption washer } \\
\hline & & Alkaline washer & $\begin{array}{c}\text { Washer after digestion } \\
\text { with } \mathrm{HCl}\end{array}$ & $\begin{array}{l}\text { Washer after phosphate } \\
\text { coating }\end{array}$ & $\begin{array}{l}\text { Washer after } \\
\text { passivation }\end{array}$ \\
\hline $\mathrm{pH}$ & - & $10.4 \pm 0.2$ & $3.06 \pm 1$ & $3.2 \pm 0.5$ & $3.85 \pm 0.5$ \\
\hline Conductivity & $\mu \mathrm{S} / \mathrm{cm}$ & $636 \pm 50$ & $861 \pm 110$ & $961 \pm 150$ & $115 \pm 44$ \\
\hline Ammonium nitrogen & $\mathrm{mg} / \mathrm{l}$ & $3.35 \pm 0.4$ & $0.102 \pm 0.03$ & $0.096 \pm 0.01$ & $0.025 \pm 0.005$ \\
\hline Nitrate $\mathrm{NO}_{3}^{-}$ & $\mathrm{mg} / \mathrm{l}$ & $5.0 \pm 0.5$ & $4.0 \pm 0.34$ & $106.0 \pm 7.0$ & $15.0 \pm 2.5$ \\
\hline Nitrite $\mathrm{NO}_{2}^{-}$ & $\mathrm{mg} / \mathrm{l}$ & $<0.02$ & $<0.02$ & $<0.02$ & $<0.02$ \\
\hline Phosphate $\mathrm{PO}_{4}^{3-}$ & $\mathrm{mg} / \mathrm{l}$ & $14.2 \pm 0.5$ & $7.96 \pm 3$ & $118.2 \pm 30$ & $20.05 \pm 5$ \\
\hline Chlorides & $\mathrm{mg} / \mathrm{l}$ & $24 \pm 4.0$ & $438 \pm 60$ & $32 \pm 7$ & $27 \pm 5$ \\
\hline Fluorides & $\mathrm{mg} / \mathrm{l}$ & $0.017 \pm 0.002$ & $0.023 \pm 0.002$ & $0.016 \pm 0.005$ & $0.256 \pm 0.1$ \\
\hline Sulphureous & $\mathrm{mg} / \mathrm{l}$ & $25 \pm 2.0$ & $22 \pm 4.0$ & $21 \pm 4.0$ & $26 \pm 1.0$ \\
\hline Alkalinity & $\mathrm{mg} / 1 \mathrm{CaCO}_{3}$ & $180 \pm 10$ & - & - & - \\
\hline $\mathrm{Na}$ & $\mathrm{mg} / \mathrm{l}$ & $345 \pm 60$ & $25 \pm 5.0$ & $78 \pm 9$ & $122 \pm 30$ \\
\hline $\mathrm{K}$ & $\mathrm{mg} / \mathrm{l}$ & $325 \pm 70$ & $33 \pm 6.0$ & $122 \pm 10$ & $24 \pm 8.0$ \\
\hline $\mathrm{Zr}$ & $\mathrm{mg} / \mathrm{l}$ & $0.022 \pm 0.01$ & $0.019 \pm 0.005$ & $0.011 \pm 0.002$ & $0.961 \pm 0.2$ \\
\hline $\mathrm{Fe}$ & $\mathrm{mg} / \mathrm{l}$ & $0.58 \pm 0.07$ & $35.5 \pm 11$ & $6.4 \pm 2.2$ & $0.54 \pm 0.1$ \\
\hline $\mathrm{Mn}$ & $\mathrm{mg} / \mathrm{l}$ & $3.0 \pm 0.2$ & $14.0 \pm 1$ & $160.0 \pm 20$ & $15.0 \pm 1.7$ \\
\hline $\mathrm{Cr}$ & $\mathrm{mg} / \mathrm{l}$ & $0.039 \pm 0.001$ & $0.005 \pm 0.001$ & $0.026 \pm 0.006$ & $0.008 \pm 0.002$ \\
\hline
\end{tabular}


Table 2. Physico-chemical composition of demineralized water and tap water. Data averaged on the basis of 6-month studies, i.e. 120 trials.

\begin{tabular}{|c|c|c|c|c|c|}
\hline No. & Parameter & Unit & Demineralized water & Tap water & Analytical method \\
\hline 1 & $\mathrm{pH}$ & - & $6.8-7.1\left(25^{\circ} \mathrm{C}\right)$ & $7.2-7.5\left(25^{\circ} \mathrm{C}\right)$ & PN-EN ISO 10523:2012 \\
\hline 2 & Conductivity & $\mu \mathrm{S} / \mathrm{cm}$ & $\begin{array}{l}\operatorname{maximum} \\
\left(25^{\circ} \mathrm{C}\right)\end{array}$ & Maximum $350\left(25^{\circ} \mathrm{C}\right)$ & PN-EN 27888:1999 \\
\hline 3 & Ammonium nitrogen & $\mathrm{mg} / \mathrm{l}$ & 0 & Maximum 0.035 & PC-C-04576-4 \\
\hline 4 & Chlorides & $\mathrm{mg} / \mathrm{l}$ & 0 & Maximum 45 & PN-ISO 9297:1994 \\
\hline 5 & Sulphureous & $\mathrm{mg} / \mathrm{l}$ & 0 & Maximum 25 & PN-79-C-04566.10 \\
\hline 6 & Total Fe & $\mathrm{mg} / \mathrm{l}$ & 0 & Maximum 0.025 & PN-EN ISO11885:2009 \\
\hline 7 & $\mathrm{Cr}(\mathrm{VI})$ & $\mu \mathrm{g} / 1$ & 0 & $<1.0$ & $\mathrm{PN}-77 / \mathrm{C} 04604 / 08$ \\
\hline 8 & Total $\mathrm{Cr}$ & $\mathrm{mg} / \mathrm{l}$ & 0 & 0.0 & $\mathrm{PN}-77 / \mathrm{C} 04604 / 08$ \\
\hline 9 & Hardness & $\mathrm{mmol} / \mathrm{l}$ & 0 & Maximum 1.50 & PN-EN ISO 11885:2009 \\
\hline 10 & $\mathrm{Mn}$ & $\mathrm{mg} / \mathrm{l}$ & 0 & Maximum 0.025 & PN-EN ISO 11885:2009 \\
\hline 11 & $\mathrm{NO}_{3}^{-}$ & $\mathrm{mg} / \mathrm{l}$ & 0 & Maximum 0.6 & PN-EN ISO 13395:2001 \\
\hline 12 & $\mathrm{NO}_{2}^{-}$ & $\mathrm{mg} / \mathrm{l}$ & 0 & Maximum 0.05 & PN-EN ISO 13395:2001 \\
\hline 13 & $\mathrm{PO}_{4}^{3-}$ & $\mathrm{mg} / \mathrm{l}$ & 0 & Maximum 0.25 & PN-EN ISO 15681:2009 \\
\hline
\end{tabular}

phosphorus compounds. Such wastewater may also contain manganese compounds (including: manganese hydrogen phosphate $\mathrm{MnHPO}_{4}$ ) [33]. Another type of sewage is wastewater and washings from the passivation of metal surfaces. Depending on the agent used, the wastewater may contain manganese, titanium, chromium, zirconium and nitric acid compounds. Due to the highly toxic, carcinogenic nature, chromium(VI) compounds are no longer used in the passivation stage of conversion coatings. Wastewaters from the manganese phosphate coating line go to one tank, where they enter into a partial reaction with one another. Next, they are pneumatically transported to the feeding tank before the neutralization process. From there, they reach the coagulation chamber, where iron(III) chloride is dosed together with sulfuric(VI) acid as a $\mathrm{pH}$ correcting substance. The next stage is the neutralization chamber, where the limewash solution is dosed, and from there it goes through the overflow to the flocculation chamber. Each reaction chamber is equipped with a mechanically driven agitator. The chambers are equipped with level and $\mathrm{pH}$ meters. The next stage of treatment is the transfer of sewage containing sludge floc to a settling tank with lamellar filling, where the flowing solid particles are separated from the sewage by gravity. The sludge is partially thickened and the surplus is pumped to the conical settling tank from where it is sent to the filter press, where the already treated sewage is completely separated from the sludge (Fig. 1) [33-34]. A schematic record of the reactions that take place during wastewater treatment can be divided into two stages, i.e. $\mathrm{pH}$ up to 9.5 and $\mathrm{pH}$ above 10 :
Reactions taking place at a $\mathrm{pH}>9.5$ :

$$
\begin{gathered}
3 \mathrm{Ca}(\mathrm{OH})_{2}+2 \mathrm{FeCl}_{3} \rightarrow 2 \mathrm{Fe}(\mathrm{OH})_{3} \downarrow+3 \mathrm{CaCl}_{2} \text { (1) } \\
\mathrm{Ca}(\mathrm{OH})_{2}+\mathrm{FeCl}_{2} \rightarrow \mathrm{Fe}(\mathrm{OH})_{2} \downarrow+\mathrm{CaCl}_{2}
\end{gathered}
$$

$5 \mathrm{Ca}(\mathrm{OH})_{2}+3 \mathrm{H}_{3} \mathrm{PO}_{4} \rightarrow \mathrm{Ca}_{5}\left(\mathrm{PO}_{4}\right)_{3} \mathrm{OH} \downarrow+9 \mathrm{H}_{2} \mathrm{O}(3)$

$$
\begin{gathered}
\mathrm{Ca}(\mathrm{OH})_{2}+2 \mathrm{HNO}_{3} \rightarrow \mathrm{Ca}\left(\mathrm{NO}_{3}\right)_{2}+2 \mathrm{H}_{2} \mathrm{O} \\
\mathrm{Ca}(\mathrm{OH})_{2}+\mathrm{H}_{2} \mathrm{ZrF}_{6} \rightarrow \mathrm{CaZrF}_{6} \downarrow+2 \mathrm{H}_{2} \mathrm{O} \\
2 \mathrm{Ca}(\mathrm{OH})_{2}+2 \mathrm{Mn}\left(\mathrm{NO}_{3}\right)_{2}+\mathrm{O}_{2} \rightarrow 2 \\
\mathrm{MnO}_{2} \cdot \mathrm{H}_{2} \mathrm{O} \downarrow+2 \mathrm{Ca}\left(\mathrm{NO}_{3}\right)_{2}
\end{gathered}
$$

Reactions taking place at a $\mathrm{pH}>10.0$ :

$$
\mathrm{Ca}(\mathrm{OH})_{2}+\mathrm{Ni}\left(\mathrm{NO}_{3}\right)_{2} \rightarrow \mathrm{Ni}(\mathrm{OH})_{2} \downarrow+\mathrm{Ca}\left(\mathrm{NO}_{3}\right)_{2}
$$

Manganese phosphate coating for rinsing of phosphate steel elements involves the use of demineralized water produced on site using ion exchange with an ion exchange resin and tap water.

The typical composition of tap water and demineralized water is shown in Table 2.

The washings stored in the buffer tank form the socalled raw sewage. A typical composition of raw sewage from manganese phosphate coating, which comprises a mixture of washings from degreasing, etching, phosphating and passivation processes, is presented in Table 3. 
Table 3. Composition of raw sewage from manganese phosphate coating.

\begin{tabular}{|c|c|c|c|c|}
\hline No. & Parameter & Result & Analytical method & Legal limit* \\
\hline 1 & $\mathrm{pH}$ & $5.9 \pm 0.5\left(21.8^{\circ} \mathrm{C}\right)$ & PN-EN ISO 10523:2012 & $6.5-9.5$ \\
\hline 2 & Conductivity & $486 \pm 33 \mu \mathrm{S} / \mathrm{cm}\left(21.8^{\circ} \mathrm{C}\right)$ & PN-EN 27888:1999 & $1500 \mu \mathrm{S} / \mathrm{cm}$ \\
\hline 3 & Ammonium nitrogen & $1.16 \pm 0.2 \mathrm{mg} / \mathrm{l}$ & Test Merck Nr 1.14752 & $200 \mathrm{mg} / \mathrm{l}$ \\
\hline 4 & Total Phosphorus & $30.5 \pm 3.0 \mathrm{mg} / 1$ & Test Merck Nr 1.14729 & $20 \mathrm{mg} / \mathrm{l}$ \\
\hline 5 & ChZT-Cr & $68 \pm 10 \mathrm{mg} / 1$ & PN-ISO 15705:2005 & $2000 \mathrm{mg} / 1$ \\
\hline 6 & $\mathrm{BZT}_{5}$ & $28 \pm 5 \mathrm{mg} / \mathrm{l}$ & PN-EN 1899-1:2002 & $1500 \mathrm{mg} / \mathrm{l}$ \\
\hline 8 & Chlorides & $70 \pm 10 \mathrm{mg} / \mathrm{l}$ & Test Merck Nr 1.14897 & $1000 \mathrm{mg} / 1$ \\
\hline 9 & Sulphureous & $25 \pm 2 \mathrm{mg} / \mathrm{l}$ & Test Merck Nr 1.00617 & $500 \mathrm{mg} / 1$ \\
\hline 10 & Total Fe & $6.63 \pm 1.0 \mathrm{mg} / 1$ & PN-EN ISO 11885:2009 & $10 \mathrm{mg} / \mathrm{l}$ \\
\hline 11 & Mo & $<0.004 \mathrm{mg} / 1$ & PN-EN ISO 11885:2009 & $1.0 \mathrm{mg} / 1$ \\
\hline 12 & $\mathrm{Cr}(\mathrm{VI})$ & $<0.010 \mathrm{mg} / 1$ & $\mathrm{PN}-77 / \mathrm{C} 04604 / 08$ & $0.1 \mathrm{mg} / 1$ \\
\hline 13 & Total $\mathrm{Cr}$ & $0.043 \pm 0.008 \mathrm{mg} / \mathrm{l}$ & PN-EN ISO 11885:2009 & $0.5 \mathrm{mg} / 1$ \\
\hline 14 & Hardness & $59.1 \pm 5 \mathrm{mg} / 1 \mathrm{CaCO}_{3}$ & PN-EN ISO 11885:2009 & - \\
\hline 15 & $\mathrm{Zn}$ & $0.203 \pm 0.06 \mathrm{mg} / 1$ & PN-EN ISO 11885 & $5.0 \mathrm{mg} / \mathrm{l}$ \\
\hline 16 & $\mathrm{Cd}$ & $<0.0005 \mathrm{mg} / 1$ & PN-EN ISO 11885:2009 & $0.4 \mathrm{mg} / \mathrm{l}$ \\
\hline 17 & $\mathrm{Mn}$ & $31.7 \pm 7 \mathrm{mg} / \mathrm{l}$ & PN-EN ISO 11885:2009 & - \\
\hline 18 & $\mathrm{Ni}$ & $0.963 \pm 0.2 \mathrm{mg} / \mathrm{l}$ & PN-EN ISO 11885:2009 & $0.5 \mathrm{mg} / 1$ \\
\hline 19 & $\mathrm{Co}$ & $0.0042 \pm 0.002 \mathrm{mg} / 1$ & PN-EN ISO 11885:2009 & $1.0 \mathrm{mg} / 1$ \\
\hline 20 & $\mathrm{~K}$ & $33.2 \pm 6 \mathrm{mg} / 1$ & PN-EN ISO 11885:2009 & $80 \mathrm{mg} / \mathrm{l}$ \\
\hline 21 & $\mathrm{Na}$ & $22.2 \pm 7.0 \mathrm{mg} / 1$ & PN-EN ISO 11885:2009 & $800 \mathrm{mg} / 1$ \\
\hline 22 & $\mathrm{NO}_{3}^{-}$ & $26.3 \pm 5.0 \mathrm{mg} / 1$ & PN-EN ISO 13395:2001 & - \\
\hline 23 & $\mathrm{NO}_{2}^{-}$ & $8.51 \pm 1.5 \mathrm{mg} / 1$ & PN-EN ISO 13395:2001 & $200 \mathrm{mg} / 1$ \\
\hline 24 & $\mathrm{~F}^{-}$ & $1.04 \pm 0.3 \mathrm{mg} / 1$ & $\mathrm{PN}-78 / \mathrm{C}-04588 / 03$ & $25.0 \mathrm{mg} / 1$ \\
\hline 25 & Volatile phenols & $0.017 \pm 0.004 \mathrm{mg} / 1$ & PN-ISO 6439:1994 & $0.1 \mathrm{mg} / \mathrm{l}$ \\
\hline 26 & TOC & $23.3 \pm 3 \mathrm{mg} / \mathrm{l}$ & PN-EN 1484:1999 & $1000 \mathrm{mg} / \mathrm{l}$ \\
\hline 27 & Total PAHs (Polycyclic aromatic hydrocarbons) & $<0.006 \mu \mathrm{g} / 1$ & PN-EN ISO 17993:2005 & $0.2 \mathrm{mg} / 1$ \\
\hline 28 & Ether extract & $15.0 \pm 4 \mathrm{mg} / \mathrm{l}$ & PN-86/C-04573.01 & $100 \mathrm{mg} / 1$ \\
\hline 29 & BTX & $<1.0 \mu \mathrm{g} / 1$ & PN-ISO 11423-1:2002 & $1.0 \mathrm{mg} / \mathrm{l}$ \\
\hline 30 & Anionic detergents ASPC & $0.73 \pm 0.2 \mathrm{mg} / 1$ & PN-EN 7875-2:2002 & $15 \mathrm{mg} / \mathrm{l}$ \\
\hline 31 & Nonionic detergents NSPC & $1.86 \pm 0.25 \mathrm{mg} / \mathrm{l}$ & PN-EN ISO 7875-2:2002 & $20 \mathrm{mg} / \mathrm{l}$ \\
\hline 32 & $\mathrm{Si}$ & $4.09 \pm 0.5 \mathrm{mg} / 1$ & PN-EN ISO 11885:2009 & - \\
\hline 33 & $\gamma$-hexachlorocyclohexane & $<0.001 \mu \mathrm{g} / 1$ & PN-EN ISO 6468:2002 & 0.0 \\
\hline 34 & Alachlor & $<0.001 \mathrm{ng} / 1$ & PN-EN ISO 6468:2002 & 0.0 \\
\hline 35 & Heptachlor & $<0.0002 \mu \mathrm{g} / 1$ & PN-EN ISO 6468:2002 & 0.0 \\
\hline 36 & Aldrin & $<0.001 \mu \mathrm{g} / 1$ & PN-EN ISO 6468:2002 & 0.0 \\
\hline 37 & Heptachlor-endo-epoxide isomer A & $<0.0002 \mathrm{ng} / 1$ & PN-EN ISO 6468:2002 & - \\
\hline 38 & Heptachlor-endo-epoxide isomer B & $<0.0002 \mathrm{ng} / 1$ & PN-EN ISO 6468:2002 & - \\
\hline 39 & Dieldrin & $<0.001 \mu \mathrm{g} / 1$ & PN-EN ISO 6468:2002 & 0.0 \\
\hline 40 & Endrin & $<0.001 \mu \mathrm{g} / 1$ & PN-EN ISO 6468:2002 & 0.0 \\
\hline
\end{tabular}


Table 3. Continued.

\begin{tabular}{|l|c|c|c|c|}
\hline 41 & Methoxychlor & $<0.001 \mu \mathrm{g} / 1$ & PN-EN ISO 6468:2002 & 0.0 \\
\hline 42 & Hexachlorobenzene & $<0.001 \mu \mathrm{g} / 1$ & PN-EN ISO 6468:2002 & $2.0 \mathrm{mg} / 1$ \\
\hline 43 & $\mathrm{Zr}$ & $0.416 \pm 0.12 \mathrm{mg} / 1$ & PN-EN ISO 11885:2009 & - \\
\hline 44 & $\mathrm{PO}_{4}^{3-}$ & $38.1 \pm 7 \mathrm{mg} / 1$ & PN-EN ISO 15681:2009 & $20.5 \mathrm{mg} / 1$ \\
\hline 45 & $\mathrm{Ca}$ & $21.7 \pm 4 \mathrm{mg} / 1$ & PN-EN ISO 11885:2009 & - \\
\hline 46 & $\mathrm{Mg}$ & $1.19 \pm 0.3 \mathrm{mg} / 1$ & PN-EN ISO 11885:2009 & - \\
\hline 47 & $\mathrm{Hg}$ & $<0.00001 \mathrm{mg} / 1$ & PN-EN ISO 11885:2009 & $0.06 \mathrm{mg} / 1$ \\
\hline
\end{tabular}

*legal limit for wastewater discharged into the sewage system based on Polish standards [42]

\section{Effectiveness of Wastewater Treatment with the Use of Coagulation}

The effectiveness of coagulation was analyzed on raw sewage for 36 months by performing a total of 750 unit analyses. In order to increase the treatment efficiency in the coagulation process with a $40 \%(\mathrm{~m} / \mathrm{m})$ solution of iron(III) chloride, a waste $30 \%(\mathrm{~m} / \mathrm{m})$ solution of sulfuric acid was used. Sulfuric acid is used in the process of cleaning the manganese phosphate coating bath from iron(III) phosphate(V) deposits, which is formed as a by-product in the above-mentioned process. Sulfuric acid does not react with iron(III) phosphate, but dissolves it, so it can be easily filtered from the working liquid. The sulfuric acid used in this way contains large amounts of iron(III), which allowed to reduce the consumption of iron(III) chloride. Thus, the costly disposal of the abovementioned sulfuric acid was abandoned. This combination permitted the reduction of high pollution values. In order to increase the efficiency of wastewater treatment, a combined flocculation process was used. The results of wastewater treatment by coagulation with flocculation are presented in Table 4.
During the analysis of the obtained results and their comparison with the typical composition of raw sewage (Table 2), it should be indicated that coagulation allowed to obtain a high reduction of biogenic compounds, i.e. orthophosphates, nitrates and heavy metals such as iron and manganese (Table 5,6). As a result of wastewater treatment by coagulation with iron(III) chloride together with waste sulfuric(VI) acid, nitrates(V) were reduced by approx. $62 \%$, phosphates(V) by approx. $78.5 \%$, iron by approx. $53 \%$, manganese by approx. $40 \%$.

The total results of 750 tests of physico-chemical analyses of treated wastewater samples using coagulation with iron(III) chloride and waste sulfuric acid(VI) carried out from January 2017 until December 2019 are shown in Table 5.

Coagulation with iron(III) chloride allows to reduce the pollutants contained in the sewage. The high efficiency of pollution reduction means that this method is readily used by the industry. One of the by-products of this process is residue of precipitated hydroxides that must be separated from the liquid portion. It is this process that is most often used in hydraulic systems, e.g. in the form of a filter press. The amount of separated sludge formed is on average $48-54 \mathrm{~kg} /$ day.

Table 4. Results of wastewater treatment by coagulation with iron(III) chloride. Data averaged on the basis of 6-month studies, i.e. 120 trials.

\begin{tabular}{|c|c|c|c|c|c|c|c|}
\hline No. & Parameter & Unit & $\begin{array}{c}\text { Raw } \\
\text { sewage }\end{array}$ & $\begin{array}{c}\text { Treated } \\
\text { wastewater }\end{array}$ & $\begin{array}{c}\text { Average pollution } \\
\text { reduction }\end{array}$ & $\begin{array}{c}\text { Reduction } \\
{[\%]}\end{array}$ & Legal limit $^{*}$ \\
\hline 1 & $\mathrm{pH}$ & - & $9.3 \pm 0.5$ & $7.8 \pm 1.1$ & - & - & $6.5-9.5$ \\
\hline 2 & Conductivity & $\mu \mathrm{S} / \mathrm{cm}$ & $428 \pm 50$ & $425 \pm 23$ & - & - & $\mathrm{max} .1500 \mu \mathrm{S} / \mathrm{cm}$ \\
\hline 3 & Chlorides & $\mathrm{mg} / \mathrm{l}$ & $95 \pm 7$ & $72 \pm 9$ & 23 & 24.2 & $\mathrm{max} .1000 \mathrm{mg} / 1$ \\
\hline 4 & Nitrates & $\mathrm{mg} / \mathrm{l}$ & $13 \pm 3$ & $5 \pm 1$ & 8 & 61.6 & - \\
\hline 5 & Nitrites & $\mathrm{mg} / 1$ & $0.04 \pm 0.02$ & $0.04 \pm 0.01$ & - & - & $\max .10 \mathrm{mg} / 1$ \\
\hline 6 & Phosphates & $\mathrm{mg} / \mathrm{l}$ & $18.5 \pm 4$ & $2.0 \pm 0.4$ & 16.5 & 78.4 & $\mathrm{max} .25 \mathrm{mg} / 1$ \\
\hline 7 & $\mathrm{Mn}$ & $\mathrm{mg} / \mathrm{l}$ & $7.6 \pm 3$ & $4.5 \pm 1.5$ & 3.1 & 40.8 & - \\
\hline 8 & $\mathrm{Fe}$ & $\mathrm{mg} / \mathrm{l}$ & $1.30 \pm 0.25$ & $0.61 \pm 0.1$ & 0.69 & 53.08 & $\mathrm{max} .10 \mathrm{mg} / 1$ \\
\hline
\end{tabular}

*legal limit for wastewater discharged into the sewage system based on Polish standards[41] 
Table 5. Results of wastewater treatment by coagulation with iron(III) chloride. Monthly averages from 3-year studies.

\begin{tabular}{|c|c|c|c|c|c|c|c|c|c|c|c|c|c|c|}
\hline & unit & January & February & March & April & May & June & July & August & September & October & November & December & $\begin{array}{c}\text { annual } \\
\text { average }\end{array}$ \\
\hline \multicolumn{15}{|c|}{2017} \\
\hline temperature & ${ }^{\circ} \mathrm{C}$ & $21.2 \pm 1$ & $22.8 \pm 1$ & $\begin{array}{r}24.4 \\
\pm 2\end{array}$ & $\begin{array}{c}25.3 \\
\pm 3\end{array}$ & $\begin{array}{c}25.3 \\
\pm 2\end{array}$ & $\begin{array}{c}28.3 \pm \\
1.2\end{array}$ & $29.6 \pm 4$ & $\begin{array}{l}30.3 \\
\pm 5\end{array}$ & $28.7 \pm 4$ & $26.6 \pm 2$ & $26.2 \pm 1$ & $25.4 \pm 1$ & 26.3 \\
\hline $\mathrm{pH}$ & - & $5.17 \pm 0.2$ & $6.30 \pm 0.1$ & $\begin{array}{c}6.61 \\
\pm 0.05 \\
\end{array}$ & $\begin{array}{c}6.27 \pm \\
0.1 \\
\end{array}$ & $\begin{array}{c}6.13 \pm \\
0.1 \\
\end{array}$ & $\begin{array}{l}9.42 \\
\pm 0.5\end{array}$ & $8.38 \pm 0.4$ & $\begin{array}{l}7.44 \\
\pm 0.2 \\
\end{array}$ & $6.60 \pm 0.2$ & $\begin{array}{l}5.54 \\
\pm 0.1 \\
\end{array}$ & $\begin{array}{l}6.17 \\
\pm 0.1 \\
\end{array}$ & $\begin{array}{c}6.15 \\
\pm 0.2 \\
\end{array}$ & 6.73 \\
\hline conductivity & $\mu \mathrm{S} / \mathrm{cm}$ & $\begin{array}{l}4387 \\
\pm 540\end{array}$ & $\begin{array}{l}2746 \\
\pm 170\end{array}$ & $\begin{array}{l}3265 \\
\pm 230\end{array}$ & $\begin{array}{l}3319 \\
\pm 345\end{array}$ & $\begin{array}{c}3330 \pm \\
270\end{array}$ & $\begin{array}{c}1644 \pm \\
120\end{array}$ & $\begin{array}{l}1350 \\
\pm 135 \\
\end{array}$ & $\begin{array}{l}2178 \\
\pm 450 \\
\end{array}$ & $\begin{array}{l}1795 \\
\pm 220 \\
\end{array}$ & $\begin{array}{l}2627 \\
\pm 250 \\
\end{array}$ & $\begin{array}{l}2282 \\
\pm 330 \\
\end{array}$ & $\begin{array}{l}2633 \\
\pm 400 \\
\end{array}$ & 2600 \\
\hline chlorides & $\mathrm{mg} / 1$ & $\begin{array}{l}724 \\
\pm 55\end{array}$ & $415 \pm 40$ & $\begin{array}{r}497 \\
\pm 20\end{array}$ & $\begin{array}{l}649 \\
\pm 54\end{array}$ & $\begin{array}{l}546 \\
\pm 35 \\
\end{array}$ & $\begin{array}{r}122 \\
\pm 19\end{array}$ & $\begin{array}{l}173 \\
\pm 20\end{array}$ & $\begin{array}{l}221 \\
\pm 22\end{array}$ & $\begin{array}{r}169 \\
\pm 15\end{array}$ & $\begin{array}{l}255 \\
\pm 50 \\
\end{array}$ & $\begin{array}{l}254 \\
\pm 40 \\
\end{array}$ & $\begin{array}{l}345 \\
\pm 40\end{array}$ & 356 \\
\hline nitrates & $\mathrm{mg} / 1$ & $\begin{array}{l}11.2 \\
\pm 0.5\end{array}$ & $\begin{array}{l}9.75 \\
\pm 0.2\end{array}$ & $\begin{array}{l}10.0 \\
\pm 0.9\end{array}$ & $\begin{array}{c}17.0 \\
\pm 1\end{array}$ & $\begin{array}{l}11.5 \\
\pm 1.2 \\
\end{array}$ & $\begin{array}{l}10.2 \\
\pm 0.5\end{array}$ & $\begin{array}{l}13.8 \\
\pm 0.4\end{array}$ & $\begin{array}{l}11.6 \\
\pm 0.6\end{array}$ & $8.9 \pm 0.5$ & $\begin{array}{l}15.0 \\
\pm 0.2\end{array}$ & $\begin{array}{l}14.6 \\
\pm 0.4\end{array}$ & $\begin{array}{l}12.4 \\
\pm 0.3\end{array}$ & 12.1 \\
\hline nitrites & $\mathrm{mg} / 1$ & $\begin{array}{c}0.08 \\
\pm 0.02\end{array}$ & $\begin{array}{c}0.12 \\
\pm 0.05\end{array}$ & $\begin{array}{c}0.10 \\
\pm 0.02\end{array}$ & $\begin{array}{l}0.048 \\
\pm 0.01\end{array}$ & 0.046 & $\begin{array}{l}0.029 \\
\pm 0.01\end{array}$ & $\begin{array}{l}0.074 \\
\pm 0.01\end{array}$ & $\begin{array}{l}0.030 \\
\pm 0.01\end{array}$ & $\begin{array}{c}0.021 \pm \\
0.02\end{array}$ & $\begin{array}{c}0.015 \\
\pm 0.005\end{array}$ & $\begin{array}{c}0.016 \\
\pm 0.007\end{array}$ & $\begin{array}{c}0.013 \\
\pm 0.004\end{array}$ & $\begin{array}{c}0.051 \\
\pm 0.008\end{array}$ \\
\hline ammonium ion & $\mathrm{mg} / \mathrm{l}$ & $\begin{array}{l}1.21 \\
\pm 0.3\end{array}$ & $\begin{array}{l}4.69 \\
\pm 0.5\end{array}$ & $\begin{array}{l}1.43 \\
\pm 0.5\end{array}$ & $\begin{array}{c}1.06 \\
\pm 0.11\end{array}$ & $\begin{array}{l}1.53 \\
\pm 0.2\end{array}$ & $\begin{array}{l}1.44 \\
\pm 0.1\end{array}$ & $\begin{array}{l}1.33 \\
\pm 0.2\end{array}$ & $\begin{array}{l}1.20 \\
\pm 0.3\end{array}$ & $1.63 \pm 0.2$ & $\begin{array}{l}1.66 \\
\pm 0.2\end{array}$ & $\begin{array}{c}1.63 \\
\pm 0.25\end{array}$ & $\begin{array}{c}1.61 \\
\pm 0.11\end{array}$ & 1.72 \\
\hline orthophosphates & $\mathrm{mg} / 1$ & $\begin{array}{l}0.66 \\
\pm 0.2\end{array}$ & $\begin{array}{l}1.20 \\
\pm 0.3\end{array}$ & $\begin{array}{l}1.70 \\
\pm 0.1\end{array}$ & $\begin{array}{l}1.57 \\
\pm 0.2\end{array}$ & $\begin{array}{l}1.76 \\
\pm 0.1\end{array}$ & $\begin{array}{l}2.54 \\
\pm 0.2\end{array}$ & $\begin{array}{l}3.73 \\
\pm 0.3\end{array}$ & $\begin{array}{l}6.93 \\
\pm 0.6\end{array}$ & $\begin{array}{c}1.67 \\
\pm 0.07\end{array}$ & $\begin{array}{l}3.48 \\
\pm 0.9\end{array}$ & $\begin{array}{l}5.11 \\
\pm 0.7\end{array}$ & $\begin{array}{c}2.15 \\
\pm 0.06\end{array}$ & 2.70 \\
\hline sulphureous & $\mathrm{mg} / 1$ & $\begin{array}{c}977.8 \\
\pm 9\end{array}$ & $\begin{array}{l}713.9 \\
\pm 20\end{array}$ & $\begin{array}{l}998.0 \\
\pm 55\end{array}$ & $\begin{array}{l}776.3 \\
\pm 50\end{array}$ & $\begin{array}{c}750.7 \\
\pm 65\end{array}$ & $\begin{array}{l}604.9 \\
\pm 40\end{array}$ & $\begin{array}{l}487.8 \\
\pm 45\end{array}$ & $\begin{array}{l}677.1 \\
\pm 60\end{array}$ & $\begin{array}{l}867.0 \\
\pm 45\end{array}$ & $\begin{array}{l}961.5 \\
\pm 120\end{array}$ & $\begin{array}{l}779.4 \\
\pm 55\end{array}$ & $\begin{array}{l}760.3 \\
\pm 60\end{array}$ & 779.2 \\
\hline $\mathrm{Fe}$ & $\mathrm{mg} / \mathrm{l}$ & $\begin{array}{l}0.76 \\
\pm 0.4 \\
\end{array}$ & $\begin{array}{l}0.35 \\
\pm 0.1 \\
\end{array}$ & $\begin{array}{c}0.40 \\
\pm 0.11 \\
\end{array}$ & $\begin{array}{l}5.11 \\
\pm 1.1 \\
\end{array}$ & $\begin{array}{c}12.08 \\
\pm 2 \\
\end{array}$ & $\begin{array}{l}0.36 \\
\pm 0.1 \\
\end{array}$ & $\begin{array}{l}0.31 \\
\pm 0.1 \\
\end{array}$ & $\begin{array}{c}0.34 \\
\pm 0.15\end{array}$ & $\begin{array}{c}0.50 \\
\pm 0.25\end{array}$ & $\begin{array}{l}0.81 \\
\pm 0.4 \\
\end{array}$ & $\begin{array}{c}0.88 \\
\pm 0.35 \\
\end{array}$ & $\begin{array}{l}1.04 \\
\pm 0.2 \\
\end{array}$ & 1.94 \\
\hline $\mathrm{Mn}$ & $\mathrm{mg} / 1$ & $\begin{array}{l}3.51 \\
\pm 0.1 \\
\end{array}$ & $\begin{array}{r}3.09 \\
\pm 0.8\end{array}$ & $\begin{array}{c}3.4 \\
\pm 0.9\end{array}$ & $\begin{array}{l}1.90 \\
\pm 0.6\end{array}$ & $\begin{array}{c}1.5 \\
\pm 0.6 \\
\end{array}$ & $\begin{array}{l}2.92 \\
\pm 0.9\end{array}$ & $\begin{array}{l}2.51 \\
\pm 0.3 \\
\end{array}$ & $\begin{array}{l}3.04 \\
\pm 1\end{array}$ & $\begin{array}{l}1.88 \\
\pm 0.7\end{array}$ & $\begin{array}{c}1.22 \\
\pm 0.24\end{array}$ & $\begin{array}{l}3.41 \\
\pm 0.7\end{array}$ & $\begin{array}{l}0.90 \\
\pm 0.4\end{array}$ & 2.44 \\
\hline $\mathrm{K}$ & $\mathrm{mg} / 1$ & $\begin{array}{l}18.75 \\
\pm 0.5\end{array}$ & $\begin{array}{l}15.9 \\
\pm 1\end{array}$ & $\begin{array}{l}25.9 \\
\pm 2\end{array}$ & $\begin{array}{l}20.6 \\
\pm 1\end{array}$ & $\begin{array}{l}15.3 \\
\pm 2 \\
\end{array}$ & $\begin{array}{l}16.0 \\
\pm 3\end{array}$ & $\begin{array}{c}13.2 \\
\pm 2\end{array}$ & $\begin{array}{r}16.9 \\
\pm 1.1 \\
\end{array}$ & $\begin{array}{l}18.9 \\
\pm 1.3 \\
\end{array}$ & $\begin{array}{l}15.7 \\
\pm 1.7 \\
\end{array}$ & $\begin{array}{l}18.3 \\
\pm 0.8\end{array}$ & $\begin{array}{l}21.8 \\
\pm 0.6\end{array}$ & 17.96 \\
\hline \multicolumn{15}{|c|}{2018} \\
\hline temperature & ${ }^{\circ} \mathrm{C}$ & $\begin{array}{l}26.0 \\
\pm 2\end{array}$ & $\begin{array}{l}25.7 \\
\pm 5\end{array}$ & $\begin{array}{c}25.4 \\
\pm 6\end{array}$ & $\begin{array}{c}26.7 \\
\pm 3\end{array}$ & $\begin{array}{c}29.4 \\
\pm 3\end{array}$ & $\begin{array}{c}29.8 \\
\pm 5\end{array}$ & $\begin{array}{l}31.0 \\
\pm 4\end{array}$ & $\begin{array}{l}29.7 \\
\pm 3\end{array}$ & $\begin{array}{l}22.6 \\
\pm 2\end{array}$ & $\begin{array}{c}22.0 \\
\pm 3\end{array}$ & $\begin{array}{c}23.0 \\
\pm 6\end{array}$ & $\begin{array}{c}23.1 \\
\pm 4\end{array}$ & 26.2 \\
\hline $\mathrm{pH}$ & - & $\begin{array}{l}5.70 \\
\pm 1.2\end{array}$ & $\begin{array}{l}7.02 \\
\pm 0.6\end{array}$ & $\begin{array}{l}8.01 \\
\pm 0.6\end{array}$ & $\begin{array}{l}9.53 \\
\pm 0.8\end{array}$ & $\begin{array}{l}10.36 \\
\pm 1.1\end{array}$ & $\begin{array}{l}8.29 \\
\pm 0.9\end{array}$ & $\begin{array}{l}9.15 \\
\pm 0.9\end{array}$ & $\begin{array}{l}7.59 \\
\pm 0.6\end{array}$ & $\begin{array}{l}7.32 \\
\pm 0.6\end{array}$ & $\begin{array}{l}7.78 \\
\pm 1.1\end{array}$ & $\begin{array}{l}8.05 \\
\pm 1.3\end{array}$ & $\begin{array}{l}8.33 \\
\pm 0.4\end{array}$ & 8.09 \\
\hline conductivity & $\mu \mathrm{S} / \mathrm{cm}$ & $\begin{array}{l}2313 \\
\pm 130\end{array}$ & $\begin{array}{l}2379 \\
\pm 220\end{array}$ & $\begin{array}{l}1983 \\
\pm 150\end{array}$ & $\begin{array}{l}2725 \\
\pm 446\end{array}$ & $\begin{array}{l}3979 \\
\pm 400\end{array}$ & $\begin{array}{c}1962 \\
\pm 430\end{array}$ & $\begin{array}{l}1791 \\
\pm 90\end{array}$ & $\begin{array}{l}1675 \\
\pm 180\end{array}$ & $\begin{array}{l}1621 \\
\pm 75\end{array}$ & $\begin{array}{c}1411 \\
\pm 140\end{array}$ & $\begin{array}{l}1555 \\
\pm 320\end{array}$ & $\begin{array}{l}1896 \\
\pm 190\end{array}$ & 2107.6 \\
\hline chlorides & $\mathrm{mg} / 1$ & $\begin{array}{l}238 \\
\pm 44\end{array}$ & $\begin{array}{l}362 \\
\pm 89\end{array}$ & $\begin{array}{c}193 \\
\pm 70\end{array}$ & $\begin{array}{l}416 \\
\pm 55\end{array}$ & $\begin{array}{l}348 \\
\pm 60\end{array}$ & $\begin{array}{l}233 \\
\pm 22\end{array}$ & $\begin{array}{l}260 \\
\pm 48\end{array}$ & $\begin{array}{l}212 \\
\pm 33\end{array}$ & $\begin{array}{l}207 \\
\pm 44\end{array}$ & $\begin{array}{l}202 \\
\pm 35\end{array}$ & $\begin{array}{l}229 \\
\pm 15\end{array}$ & $\begin{array}{l}204 \\
\pm 20\end{array}$ & 258.7 \\
\hline nitrates & $\mathrm{mg} / 1$ & $\begin{array}{l}12.8 \\
\pm 1.1\end{array}$ & $\begin{array}{l}13.2 \\
\pm 1\end{array}$ & $\begin{array}{l}11.6 \\
\pm 2.2\end{array}$ & $\begin{array}{r}11.7 \\
\pm 1.7\end{array}$ & $\begin{array}{l}17.8 \\
\pm 0.6\end{array}$ & $\begin{array}{r}16.7 \\
\pm 0.9\end{array}$ & $\begin{array}{r}14.9 \\
\pm 0.7\end{array}$ & $\begin{array}{r}13.0 \\
\pm 0.9\end{array}$ & $\begin{array}{r}13.1 \\
\pm 2.2\end{array}$ & $\begin{array}{r}11.5 \\
\pm 2.6\end{array}$ & $\begin{array}{r}12.2 \\
\pm 1.5\end{array}$ & $\begin{array}{r}10.5 \\
\pm 1.8\end{array}$ & 13.3 \\
\hline nitrites & $\mathrm{mg} / \mathrm{l}$ & $\begin{array}{l}0.026 \\
\pm 0.01 \\
\end{array}$ & $\begin{array}{c}0.019 \\
\pm 0.005 \\
\end{array}$ & $\begin{array}{r}0.088 \\
\pm 0.02 \\
\end{array}$ & $\begin{array}{l}0.054 \\
\pm 0.02\end{array}$ & $\begin{array}{l}0.063 \\
\pm 0.01\end{array}$ & $\begin{array}{l}0.129 \\
\pm 0.01\end{array}$ & $\begin{array}{l}0.063 \\
\pm 0.02\end{array}$ & $\begin{array}{l}0.213 \\
\pm 0.03 \\
\end{array}$ & $\begin{array}{l}0.145 \\
\pm 0.04 \\
\end{array}$ & $\begin{array}{c}0.186 \\
\pm 0.013 \\
\end{array}$ & $\begin{array}{l}0.129 \\
\pm 0.02\end{array}$ & $\begin{array}{l}0.435 \\
\pm 0.1\end{array}$ & 0.13 \\
\hline ammonium ion & $\mathrm{mg} / 1$ & $\begin{array}{l}1.87 \\
\pm 0.4\end{array}$ & $\begin{array}{l}1.87 \\
\pm 0.4\end{array}$ & $\begin{array}{l}2.01 \\
\pm 0.5\end{array}$ & $\begin{array}{l}2.74 \\
\pm 0.2\end{array}$ & $\begin{array}{l}2.83 \\
\pm 0.7\end{array}$ & $\begin{array}{l}2.96 \\
\pm 0.8\end{array}$ & $\begin{array}{l}3.15 \\
\pm 0.8 \\
\end{array}$ & $\begin{array}{c}2.92 \\
\pm 1\end{array}$ & $\begin{array}{l}2.61 \\
\pm 0.6 \\
\end{array}$ & $\begin{array}{l}2.80 \\
\pm 0.4\end{array}$ & $\begin{array}{l}2.91 \\
\pm 0.8 \\
\end{array}$ & $\begin{array}{l}3.28 \\
\pm 1.1 \\
\end{array}$ & 1.87 \\
\hline orthophosphates & $\mathrm{mg} / 1$ & $\begin{array}{l}2.58 \\
\pm 1.1\end{array}$ & 1.91 & 2.61 & 1.92 & 1.34 & 3.68 & 1.43 & 2.88 & 1.84 & 2.05 & 2.01 & 1.21 & 2.11 \\
\hline sulphureous & $\mathrm{mg} / 1$ & $\begin{array}{l}910.5 \\
\pm 230\end{array}$ & $\begin{array}{l}707.0 \\
\pm 120\end{array}$ & $\begin{array}{c}427.0 \\
\pm 50\end{array}$ & $\begin{array}{c}121.2 \\
\pm 55\end{array}$ & $\begin{array}{c}351.8 \\
\pm 54\end{array}$ & $\begin{array}{c}555.7 \\
\pm 40\end{array}$ & $\begin{array}{c}398.4 \\
\pm 30\end{array}$ & $\begin{array}{c}502.9 \\
\pm 25\end{array}$ & $\begin{array}{c}448.8 \\
\pm 60\end{array}$ & $\begin{array}{c}287.5 \\
\pm 45\end{array}$ & $\begin{array}{l}343.3 \\
\pm 120\end{array}$ & $\begin{array}{l}480.8 \\
\pm 55\end{array}$ & 910.5 \\
\hline $\mathrm{Fe}$ & $\mathrm{mg} / \mathrm{s}$ & $\begin{array}{l}0.870 \\
\pm 0.11\end{array}$ & $\begin{array}{l}0.18 \\
\pm 0.2\end{array}$ & $\begin{array}{l}0.719 \\
\pm 0.2\end{array}$ & $\begin{array}{l}0.139 \\
\pm 0.04\end{array}$ & $\begin{array}{l}0.117 \\
\pm 0.1\end{array}$ & $\begin{array}{l}0.472 \\
\pm 0.1\end{array}$ & $\begin{array}{l}0.298 \\
\pm 0.1\end{array}$ & $\begin{array}{c}0.32 \\
\pm 0.05\end{array}$ & $\begin{array}{l}0.34 \\
\pm 0.9\end{array}$ & $\begin{array}{c}0.31 \\
\pm 0.12\end{array}$ & $\begin{array}{c}0.21 \\
\pm 0.04\end{array}$ & $\begin{array}{r}2.06 \\
\pm 0.8 \\
\end{array}$ & 0.870 \\
\hline Mn & $\mathrm{mg} / 1$ & $\begin{array}{l}3.19 \\
\pm 0.2\end{array}$ & $\begin{array}{l}1.74 \\
\pm 0.8\end{array}$ & $\begin{array}{c}0.79 \\
\pm 0.35\end{array}$ & $\begin{array}{l}0.45 \\
\pm 0.2\end{array}$ & $\begin{array}{l}0.50 \\
\pm 0.1\end{array}$ & $\begin{array}{l}1.49 \\
\pm 0.2\end{array}$ & $\begin{array}{l}1.27 \\
\pm 0.3\end{array}$ & $\begin{array}{c}1.41 \\
\pm 0.11\end{array}$ & $\begin{array}{l}1.09 \\
\pm 0.4\end{array}$ & $\begin{array}{l}0.84 \\
\pm 0.4\end{array}$ & $\begin{array}{l}2.16 \\
\pm 0.8\end{array}$ & $\begin{array}{l}3.43 \\
\pm 0.6\end{array}$ & 3.19 \\
\hline $\mathrm{K}$ & $\mathrm{mg} / \mathrm{l}$ & $\begin{array}{l}22.0 \\
\pm 5 \\
\end{array}$ & $\begin{array}{l}18.3 \\
\pm 4 \\
\end{array}$ & $\begin{array}{l}17.7 \\
\pm 6 \\
\end{array}$ & $\begin{array}{l}21.8 \\
\pm 3 \\
\end{array}$ & $\begin{array}{l}30.3 \\
\pm 8 \\
\end{array}$ & $\begin{array}{l}20.9 \\
\pm 4.8 \\
\end{array}$ & $\begin{array}{l}19.9 \\
\pm 5 \\
\end{array}$ & $\begin{array}{l}19.1 \\
\pm 3\end{array}$ & $\begin{array}{r}19.4 \\
\pm 3.3 \\
\end{array}$ & $\begin{array}{l}19.8 \\
\pm 0.9\end{array}$ & $\begin{array}{l}18.0 \\
\pm 2.6\end{array}$ & $\begin{array}{l}20.9 \\
\pm 2.5 \\
\end{array}$ & 22.0 \\
\hline \multicolumn{15}{|c|}{2019} \\
\hline temperature & ${ }^{\circ} \mathrm{C}$ & $\begin{array}{c}24.5 \\
\pm 3\end{array}$ & $\begin{array}{l}25.2 \\
\pm 2.5\end{array}$ & $\begin{array}{c}26.9 \\
\pm 3\end{array}$ & $\begin{array}{c}27.0 \\
\pm 4\end{array}$ & $\begin{array}{c}29.6 \\
\pm 5\end{array}$ & $\begin{array}{l}29.8 \\
\pm 5\end{array}$ & $\begin{array}{c}32.2 \\
\pm 2\end{array}$ & $\begin{array}{c}31.5 \\
\pm 3\end{array}$ & $\begin{array}{c}24.3 \\
\pm 5\end{array}$ & $\begin{array}{c}23.6 \\
\pm 3\end{array}$ & $\begin{array}{l}22.6 \\
\pm 2.5\end{array}$ & $\begin{array}{c}21.6 \\
\pm 3\end{array}$ & 26.6 \\
\hline $\mathrm{pH}$ & - & $\begin{array}{l}6.60 \\
\pm 1.1 \\
\end{array}$ & $\begin{array}{r}6.90 \\
\pm 1.9\end{array}$ & $\begin{array}{l}7.50 \\
\pm 0.6\end{array}$ & $\begin{array}{l}9.20 \\
\pm 1.9\end{array}$ & $\begin{array}{l}9.30 \\
\pm 1.3 \\
\end{array}$ & $\begin{array}{c}7.50 \\
\pm 1.1\end{array}$ & $\begin{array}{l}9.20 \\
\pm 0.6\end{array}$ & $\begin{array}{r}8.20 \\
\pm 0.5\end{array}$ & $\begin{array}{r}7.90 \\
\pm 0.5\end{array}$ & $\begin{array}{l}7.10 \\
\pm 0.6\end{array}$ & $\begin{array}{c}8.60 \\
\pm 0.7\end{array}$ & $\begin{array}{l}8.40 \\
\pm 0.6\end{array}$ & 8.03 \\
\hline conductivity & $\mu \mathrm{S} / \mathrm{cm}$ & $\begin{array}{l}1225 \\
\pm 50 \\
\end{array}$ & $\begin{array}{r}1296 \\
\pm 130 \\
\end{array}$ & $\begin{array}{l}1449 \\
\pm 70\end{array}$ & $\begin{array}{l}1563 \\
\pm 60 \\
\end{array}$ & $\begin{array}{c}1775 \\
\pm 140\end{array}$ & $\begin{array}{l}1225 \\
\pm 200\end{array}$ & $\begin{array}{r}1412 \\
\pm 235 \\
\end{array}$ & $\begin{array}{r}1633 \\
\pm 55 \\
\end{array}$ & $\begin{array}{l}1425 \\
\pm 40\end{array}$ & $\begin{array}{l}1711 \\
\pm 70\end{array}$ & $\begin{array}{l}900 \\
\pm 67 \\
\end{array}$ & $\begin{array}{c}1563 \\
\pm\end{array}$ & 1431.4 \\
\hline chlorides & $\mathrm{mg} / \mathrm{l}$ & $\begin{array}{l}211 \\
\pm 30\end{array}$ & $\begin{array}{l}252 \\
\pm 44 \\
\end{array}$ & $\begin{array}{l}205 \\
\pm 40\end{array}$ & $\begin{array}{l}332 \\
\pm 20 \\
\end{array}$ & $\begin{array}{c}274 \\
\pm 28\end{array}$ & $\begin{array}{l}241 \\
\pm 35\end{array}$ & $\begin{array}{c}233 \\
\pm 40 \\
\end{array}$ & $\begin{array}{l}256 \\
\pm 55\end{array}$ & $\begin{array}{r}266 \\
\pm 30\end{array}$ & $\begin{array}{r}296 \\
\pm 40\end{array}$ & $\begin{array}{l}211 \\
\pm 20\end{array}$ & $\begin{array}{r}205 \\
\pm 25\end{array}$ & 248.5 \\
\hline nitrates & $\mathrm{mg} / 1$ & $\begin{array}{r}13.0 \\
\pm 2.4\end{array}$ & $\begin{array}{c}2.9 \\
\pm 2.2 \\
\end{array}$ & $\begin{array}{r}12.3 \\
\pm 1.9\end{array}$ & $\begin{array}{c}7.5 \\
\pm 3.4\end{array}$ & \begin{tabular}{|l|}
16.3 \\
\pm 2.2 \\
\end{tabular} & $\begin{array}{c}14.2 \\
\pm 3\end{array}$ & $\begin{array}{r}12.3 \\
\pm 2.8\end{array}$ & $\begin{array}{r}11.0 \\
\pm 1.1\end{array}$ & $\begin{array}{c}10.3 \\
\pm 2.7\end{array}$ & $\begin{array}{c}11.6 \\
\pm 3\end{array}$ & $\begin{array}{r}12.9 \\
\pm 3.9\end{array}$ & $\begin{array}{l}10.5 \\
\pm 5\end{array}$ & 11.2 \\
\hline nitrites & $\mathrm{mg} / \mathrm{l}$ & $\begin{array}{c}0.022 \\
\pm 0.04\end{array}$ & $\begin{array}{c}0.015 \\
\pm 0.01\end{array}$ & $\begin{array}{c}0.08 \\
\pm 0.01\end{array}$ & $\begin{array}{c}0.123 \\
\pm 0.04\end{array}$ & $\begin{array}{l}0.123 \\
\pm 0.2\end{array}$ & $\begin{array}{c}0.112 \\
\pm 0.01\end{array}$ & $\begin{array}{c}0.078 \\
\pm 0.03\end{array}$ & $\begin{array}{c}0.121 \\
\pm 0.01\end{array}$ & $\begin{array}{c}0.088 \\
\pm 0.03\end{array}$ & $\begin{array}{c}0.098 \\
\pm 0.04\end{array}$ & $\begin{array}{c}0.22 \\
\pm 0.03\end{array}$ & $\begin{array}{c}0.036 \\
\pm 0.04\end{array}$ & 0.082 \\
\hline ammonium ion & $\mathrm{mg} / 1$ & $\begin{array}{c}0.030 \\
\pm 0.015\end{array}$ & $\begin{array}{l}0.025 \\
\pm 0.01 \\
\end{array}$ & $\begin{array}{r}0.077 \\
\pm 0.04 \\
\end{array}$ & $\begin{array}{l}0.036 \\
\pm 0.01\end{array}$ & $\begin{array}{c}0.055 \\
\pm 0.02 \\
\end{array}$ & $\begin{array}{l}0.098 \\
\pm 0.02\end{array}$ & $\begin{array}{l}0.074 \\
\pm 0.03\end{array}$ & $\begin{array}{c}0.125 \\
\pm 0.06\end{array}$ & $\begin{array}{l}0.174 \\
\pm 0.02 \\
\end{array}$ & $\begin{array}{l}0.196 \\
\pm 0.03\end{array}$ & $\begin{array}{l}0.177 \\
\pm 0.09\end{array}$ & $\begin{array}{l}0.363 \\
\pm 0.07 \\
\end{array}$ & 0.12 \\
\hline orthophosphates & $\mathrm{mg} / \mathrm{l}$ & $\begin{array}{l}1.22 \\
\pm 0.5\end{array}$ & $\begin{array}{l}1.36 \\
\pm 0.4\end{array}$ & $\begin{array}{l}1.96 \\
\pm 0.3\end{array}$ & $\begin{array}{c}1.74 \\
\pm 0.4\end{array}$ & $\begin{array}{l}2.20 \\
\pm 0.6\end{array}$ & $\begin{array}{l}2.30 \\
\pm 0.3\end{array}$ & $\begin{array}{l}2.90 \\
\pm 0.4\end{array}$ & $\begin{array}{l}3.05 \\
\pm 0.5\end{array}$ & $\begin{array}{l}2.70 \\
\pm 0.6\end{array}$ & $\begin{array}{l}2.44 \\
\pm 0.9\end{array}$ & $\begin{array}{l}2.93 \\
\pm 0.2\end{array}$ & $\begin{array}{l}2.80 \\
\pm 0.7\end{array}$ & 2.30 \\
\hline sulphureous & $\mathrm{mg} / 1$ & $\begin{array}{l}3.30 \\
\pm 1.3\end{array}$ & $\begin{array}{l}2.30 \\
\pm 0.9\end{array}$ & $\begin{array}{l}2.80 \\
\pm 1.1\end{array}$ & $\begin{array}{l}2.50 \\
\pm 0.9\end{array}$ & $\begin{array}{l}2.30 \\
\pm 0.8\end{array}$ & $\begin{array}{l}2.70 \\
\pm 0.7\end{array}$ & $\begin{array}{l}2.99 \\
\pm 0.9\end{array}$ & $\begin{array}{l}2.90 \\
\pm 0.5\end{array}$ & $\begin{array}{l}3.50 \\
\pm 0.4\end{array}$ & $\begin{array}{l}2.20 \\
\pm 0.8\end{array}$ & $\begin{array}{l}2.90 \\
\pm 0.2\end{array}$ & $\begin{array}{l}2.70 \\
\pm 0.6\end{array}$ & 2.8 \\
\hline $\mathrm{Fe}$ & $\mathrm{mg} / 1$ & $\begin{array}{l}1.452 \\
\pm 1.1\end{array}$ & $\begin{array}{l}2.103 \\
\pm 0.09\end{array}$ & $\begin{array}{l}1.50 \\
\pm 1\end{array}$ & $\begin{array}{c}1.06 \\
\pm 0.04\end{array}$ & $\begin{array}{c}10.0 \\
0.82 \\
\pm 0.09\end{array}$ & $\begin{array}{c}0.66 \\
\pm 0.05\end{array}$ & $\begin{array}{c}1.16 \\
\pm 0.03\end{array}$ & $\begin{array}{c}0.33 \\
\pm 0.04\end{array}$ & $\begin{array}{c} \pm .19 \\
0.19 \\
\pm 0.02\end{array}$ & $\begin{array}{c}3.0 \\
\pm 0.1\end{array}$ & $\begin{array}{c}1.20 \\
\pm 0.2\end{array}$ & $\begin{array}{l}0.711 \\
\pm 0.4\end{array}$ & 1.26 \\
\hline $\mathrm{Mn}$ & $\mathrm{mg} / \mathrm{l}$ & $\begin{array}{l}2.770 \\
\pm 1.3\end{array}$ & $\begin{array}{l}2.69 \\
\pm 1.4\end{array}$ & $\begin{array}{l}3.770 \\
\pm 1.5\end{array}$ & $\begin{array}{l}1.250 \\
\pm 0.9\end{array}$ & $\begin{array}{l}3.360 \\
\pm 2.2\end{array}$ & $\begin{array}{l}2.450 \\
\pm 0.1\end{array}$ & $\begin{array}{l}1.220 \\
\pm 0.7\end{array}$ & $\begin{array}{l}3.19 \\
\pm 0.3\end{array}$ & $\begin{array}{l}3.38 \\
\pm 1.3\end{array}$ & $\begin{array}{l}3.55 \\
\pm 1.8\end{array}$ & $\begin{array}{l}3.29 \\
\pm 1.0\end{array}$ & $\begin{array}{l}3.20 \\
\pm 1.2\end{array}$ & 2.84 \\
\hline $\mathrm{K}$ & $\mathrm{mg} / \mathrm{s}$ & $\begin{array}{r}0.96 \\
\pm 0.5 \\
\end{array}$ & $\begin{array}{r}1.50 \\
\pm 1.0 \\
\end{array}$ & $\begin{array}{l}0.70 \\
\pm 0.9\end{array}$ & $\begin{array}{l}0.90 \\
\pm 0.8\end{array}$ & $\begin{array}{l}0.90 \\
\pm 0.7\end{array}$ & $\begin{array}{l}1.22 \\
\pm 0.6\end{array}$ & $\begin{array}{r}1.78 \\
\pm 0.3\end{array}$ & $\begin{array}{l}1.26 \\
\pm 0.5\end{array}$ & $\begin{array}{r}1.30 \\
\pm 0.3\end{array}$ & $\begin{array}{c}1.50 \\
\pm 0.7\end{array}$ & $\begin{array}{c}1.70 \\
\pm 0.6\end{array}$ & $\begin{array}{c}1.2 \\
2.20 \\
\pm 1.1\end{array}$ & 1.4 \\
\hline
\end{tabular}

The average degree of pollution reduction by coagulation is shown in Table 6 .

\section{Effectiveness of Wastewater Treatment with the Use of Ozonation}

The ozonation process showed that the ozone produced in the amount of $0.00235 \mathrm{~mol} / \mathrm{dm}^{3}$ reacted differently with ozonated sewage samples (treated and raw sewage). Carried out for 60 minutes, it resulted in the raw sewage's lack of initial reaction with the generated ozone; the generated ozone began to react with the sewage only from the $40^{\text {th }}$ minute.

The wastewater treated with coagulation and subjected to ozonation behaved differently than the raw sewage sample. Within 0-20 minutes, the ozone passed through the column reacted with the wastewater sample. Only after 20 minutes, its content in the KI washer 
Table 6. Average degree of pollution reduction with iron(III) chloride and waste sulfuric(VI) acid.

\begin{tabular}{|c|c|c|c|c|}
\hline & \multicolumn{4}{|c|}{ Average annual reduction [\%] } \\
\hline Year & Orthophosphate & Nitrates & Fe & $92.3 \pm 4$ \\
\hline 2017 & $92.9 \pm 5$ & $54.0 \pm 4.5$ & $70.7 \pm 4$ & $90 \pm 2.5$ \\
\hline 2018 & $94.4 \pm 4$ & $49.5 \pm 4$ & $86.8 \pm 5$ & $91.04 \pm 3$ \\
\hline 2019 & $93.4 \pm 4$ & $57.5 \pm 5$ & $81 \pm 5$ & . \\
\hline
\end{tabular}

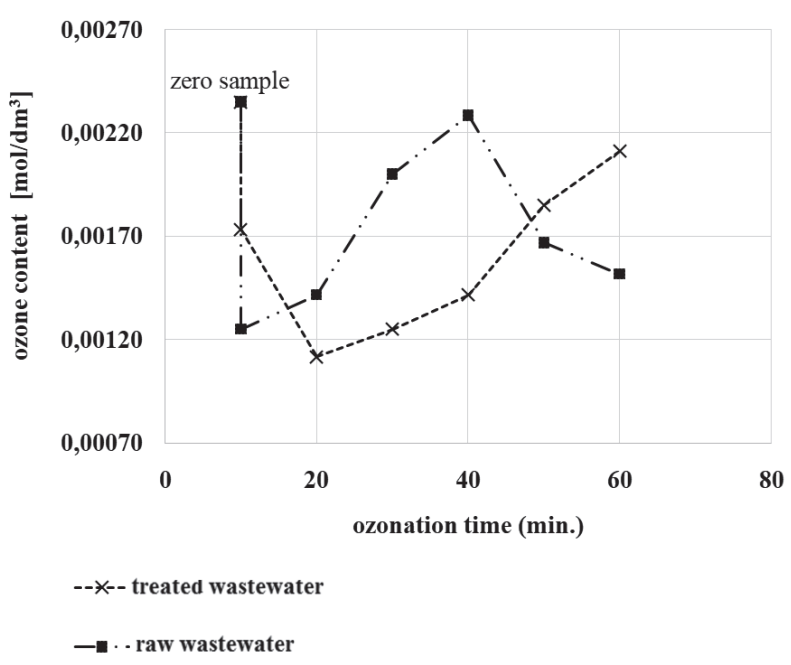

Fig. 3. Reaction of ozone with raw sewage and treated sewage by coagulation.

increased proportionally, which proves that ozonation is complete. Ozone reaction with the wastewater dose is shown in Fig. 3.

During ozonation, $5 \mathrm{ml}$ of samples were taken for UV-VIS spectrophotometric measurements. As a result of ozonation, the orthophosphate content was reduced by $28 \%$ for raw sewage and by $45 \%$ for treated sewage. The content of nitrates( $\mathrm{V})$ was reduced by $60 \%$ for raw sewage and by $20 \%$ for sewage treated with coagulation.

However, the content of nitrates in raw sewage during ozonation initially decreased to the value of 4-6 $\mathrm{mg} / \mathrm{l}$ within 20-30 min. of the process. Within 30 minutes, this value increased to $19 \mathrm{mg} / \mathrm{l}$, which proves the oxidation of all nitrogen compounds contained in the wastewater to the form of nitrates $(\mathrm{V})$, which then decreased to the value of $5.2 \mathrm{mg} / \mathrm{l}$. The results of analyses are presented in Fig. 4.

In addition to parameters such as the content of biogenic compounds, a turbidity analysis per standard unit of formazin was also performed for wastewater samples. As a result of ozonation, different results were obtained on samples treated with coagulation and on the raw sewage sample. Initially, the turbidity of the sample was $31.5 \mathrm{NTU}$, and that of the treated sewage was 29.5 NTU. With conducting ozonation, the turbidity of treated sewage was reduced to 21.6 NTU, and the turbidity of raw sewage increased to 51.6 NTU. Raw sewage contains large amounts of dissolved iron(II) and manganese(II) compounds (Table 1), which is easily oxidized to iron(III) and manganese(IV) compounds in colloidal formunder the influence of ozone, hence the observed increase in turbidity (Fig. 5).

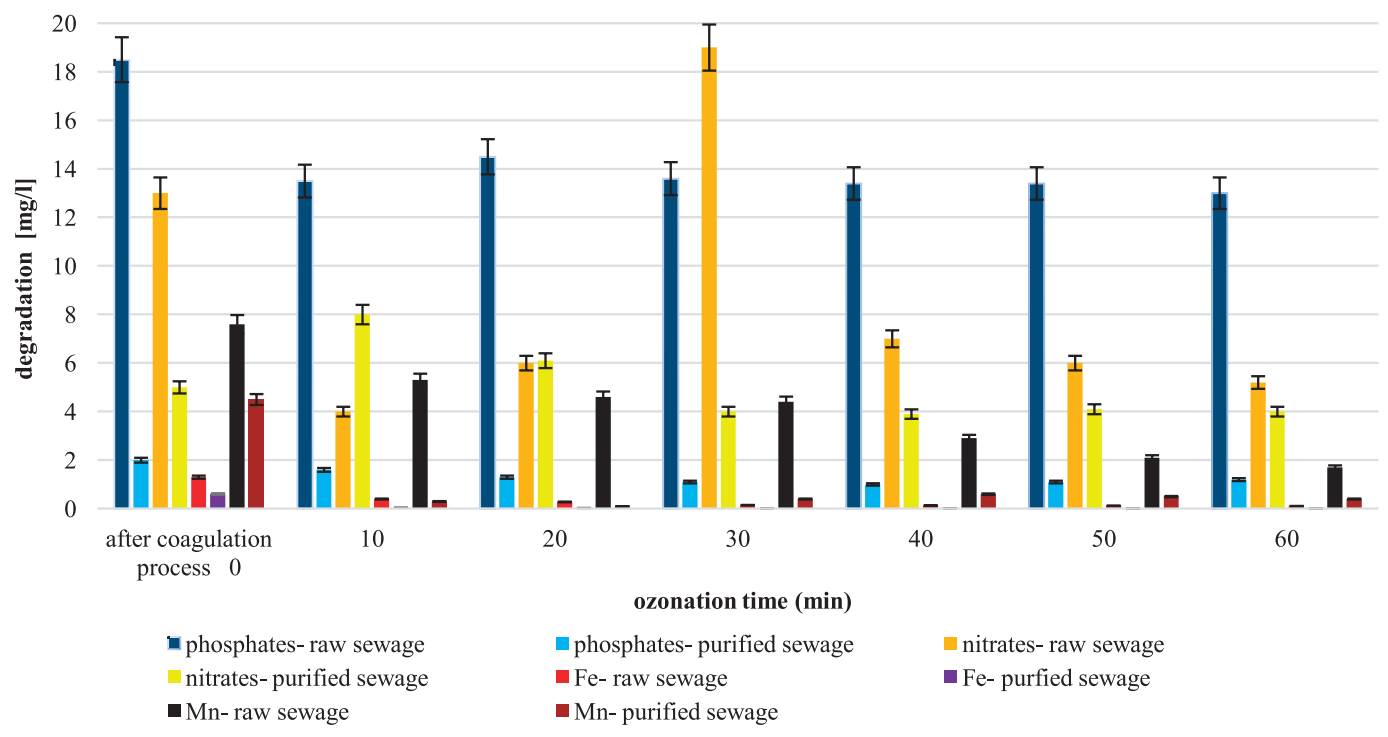

Fig. 4. Results for treated and raw sewage during ozonation. 


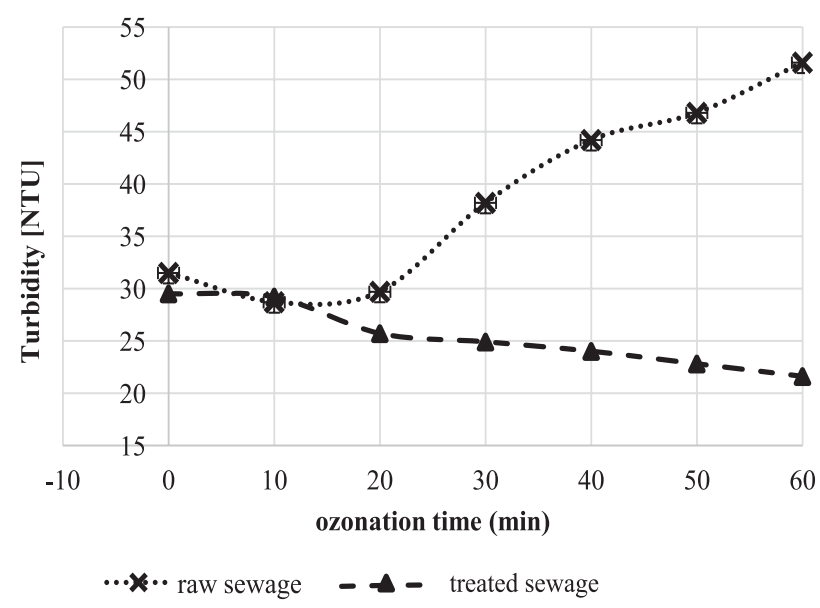

Fig. 5. Results of turbidity measurement of raw and treated sewage samples.

The sewage treated with coagulation contains minor amounts of suspended solids which are decomposed into a soluble form under the influence of ozone, hence the decrease in the value of the turbidity parameter (Fig. 5).
Table 7. Average degree of reduction of nitrates $(\mathrm{V})$ using the Fenton reaction.

\begin{tabular}{|c|c|c|}
\hline Dose of $\mathbf{H}_{\mathbf{2}} \mathbf{O}_{\mathbf{2}}(\mathbf{m l})$ & $\mathbf{2}$ & $\mathbf{3}$ \\
\hline Reaction time (min.) & \multicolumn{2}{|c|}{ Concentration of nitrates $(\mathrm{mg} / \mathrm{l})$} \\
\hline $\mathbf{0}$ & 13.00 & 13.00 \\
\hline $\mathbf{1}$ & 2.20 & 3.90 \\
\hline $\mathbf{5}$ & 0.089 & 0.075 \\
\hline $\mathbf{1 0}$ & 0.075 & 0.037 \\
\hline $\mathbf{2 0}$ & 0.041 & 0.014 \\
\hline $\mathbf{3 0}$ & 0.031 & 0.012 \\
\hline
\end{tabular}

\section{Fenton Reaction}

The Fenton reaction was carried out in two variants with and without the addition of $\mathrm{FeCl}_{3}$ solution, which is dictated by the iron content in raw sewage in the amount of $1.63 \mathrm{mg} / \mathrm{l}$. The Fenton process was carried out for 30 minutes in 5 variants in $\mathrm{pH}$ 4.0-5.0: without the addition of iron with a dose of $\mathrm{H}_{2} \mathrm{O}_{2}: 1,2,3 \mathrm{ml}$ and
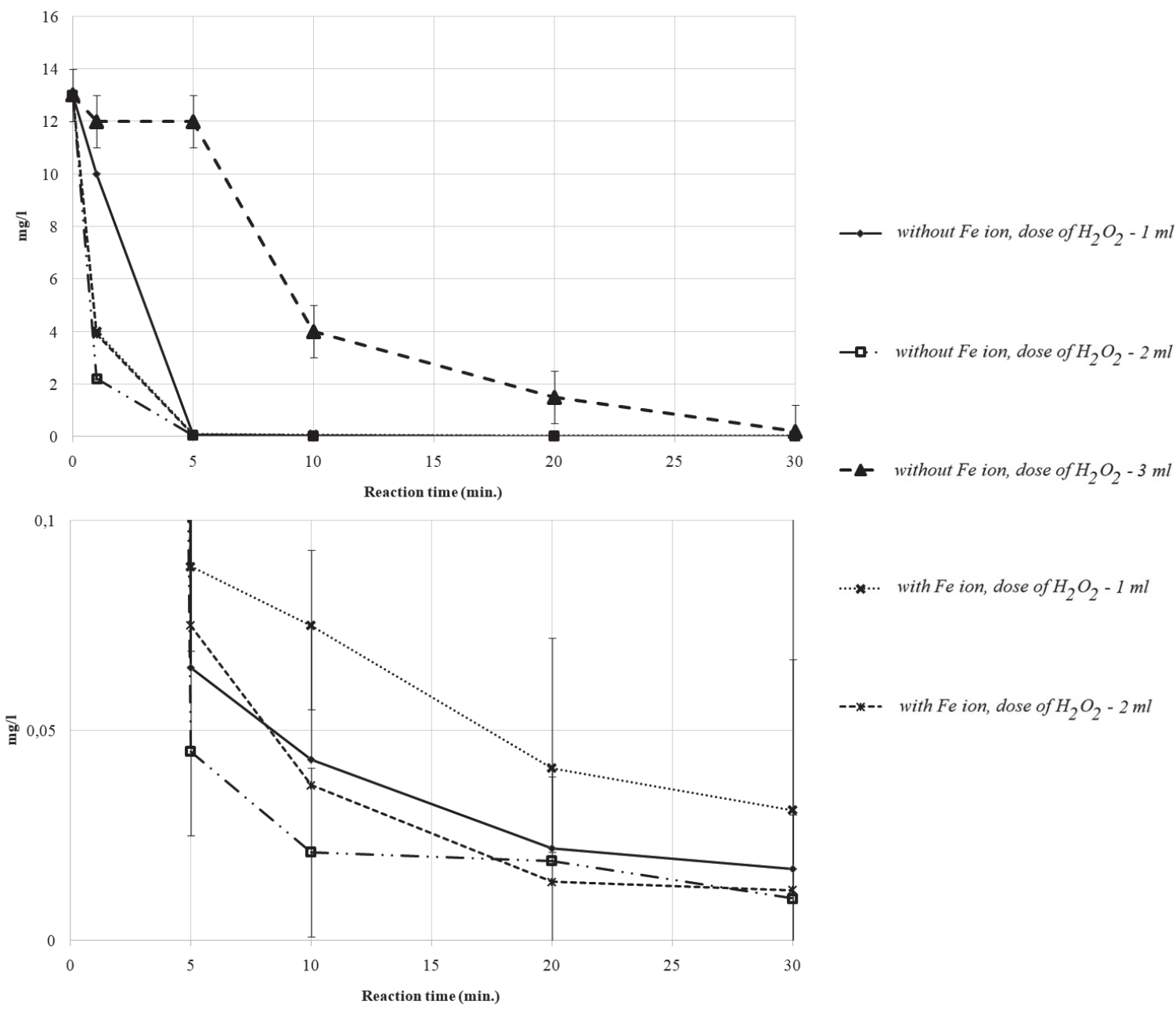

…... with Fe ion, dose of $\mathrm{H}_{2} \mathrm{O}_{2}-1 \mathrm{ml}$

--*-- with Fe ion, dose of $\mathrm{H}_{2} \mathrm{O}_{2}-2 \mathrm{ml}$

Fig. 6. Reduction of nitrate(V) content using the Fenton method. 


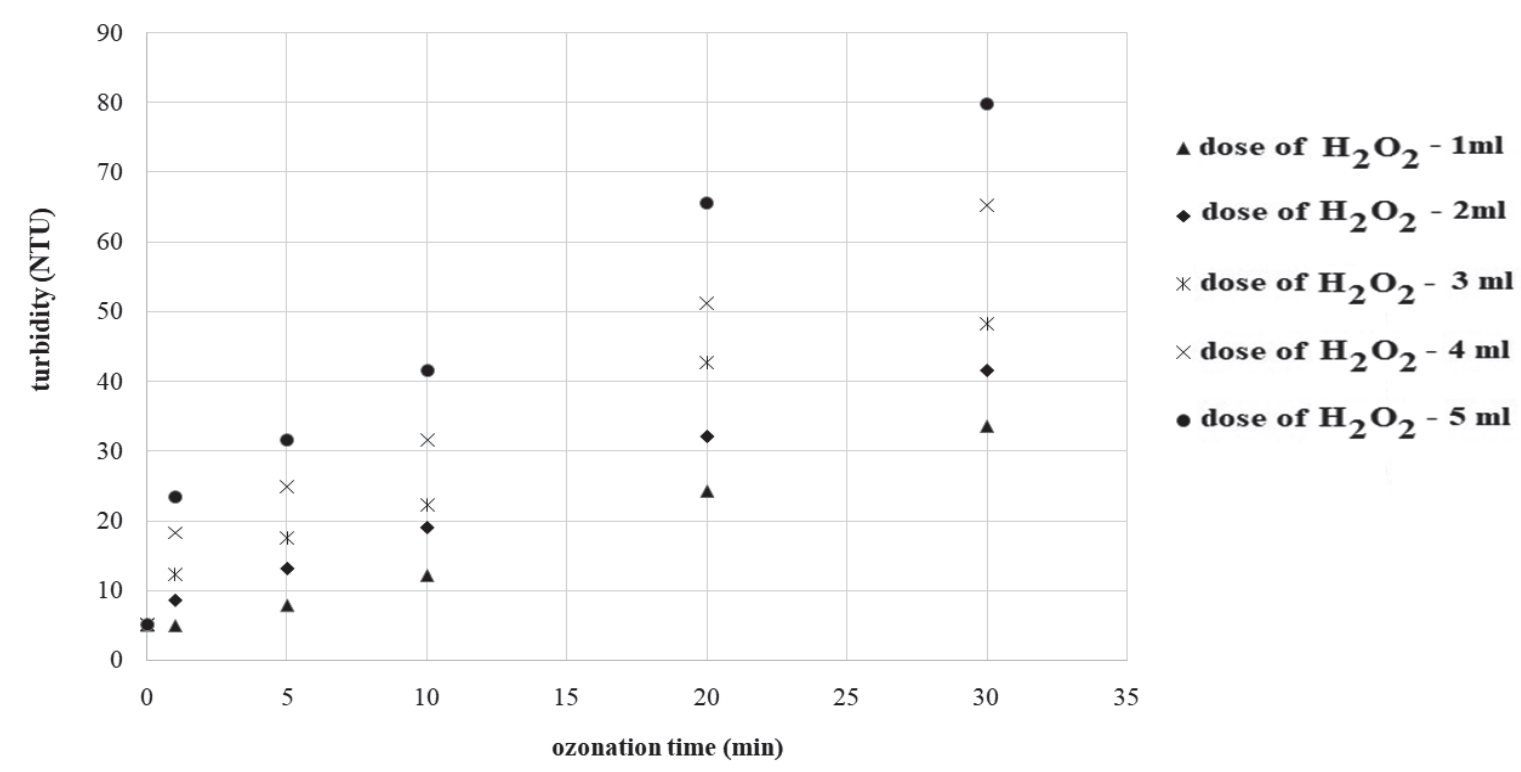

Fig. 7. Determination of turbidity in raw sewage samples depending on the dose of hydrogen peroxide.
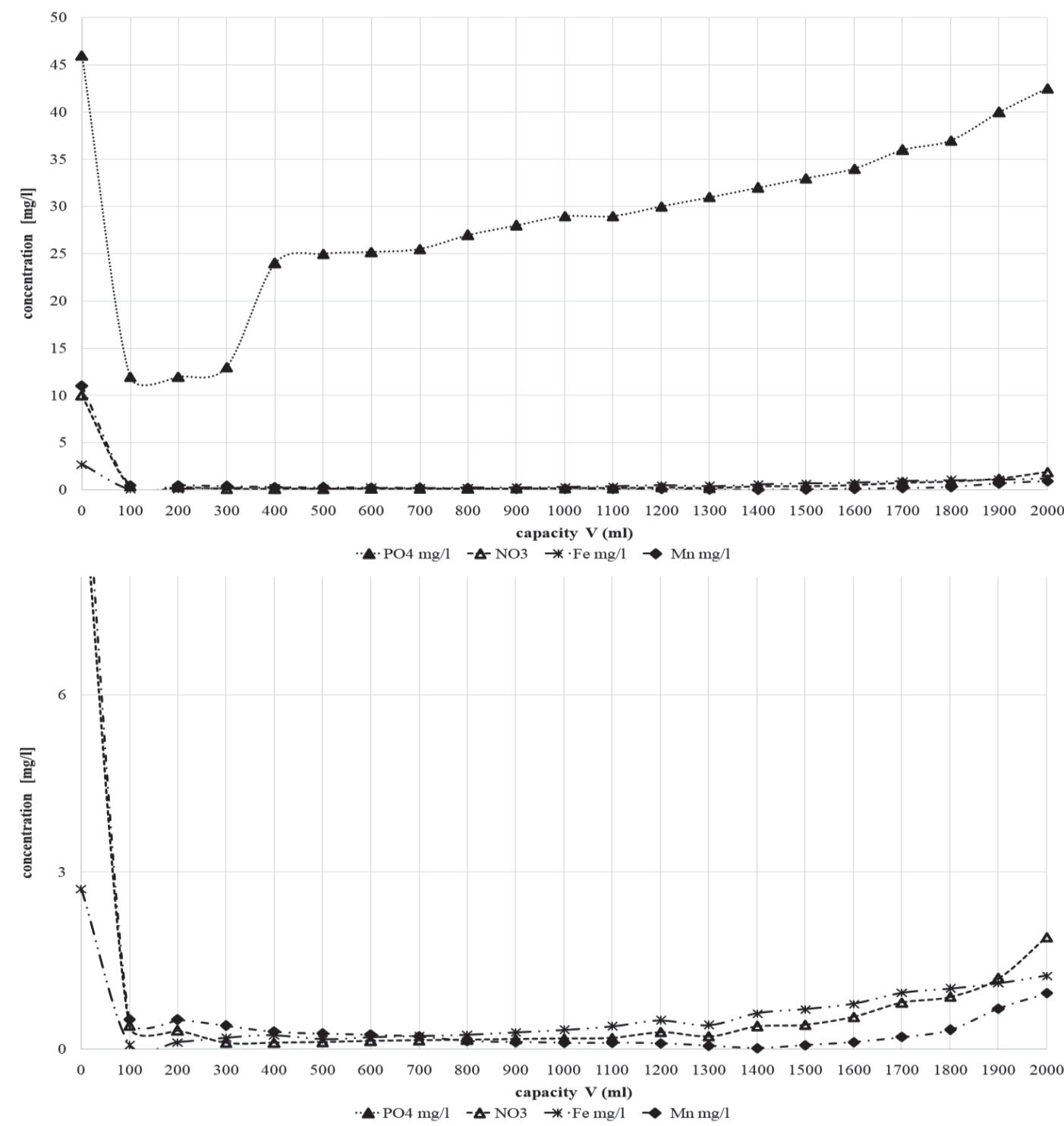

Fig. 8. Wastewater sorption using the activated carbon-zeolite system. 


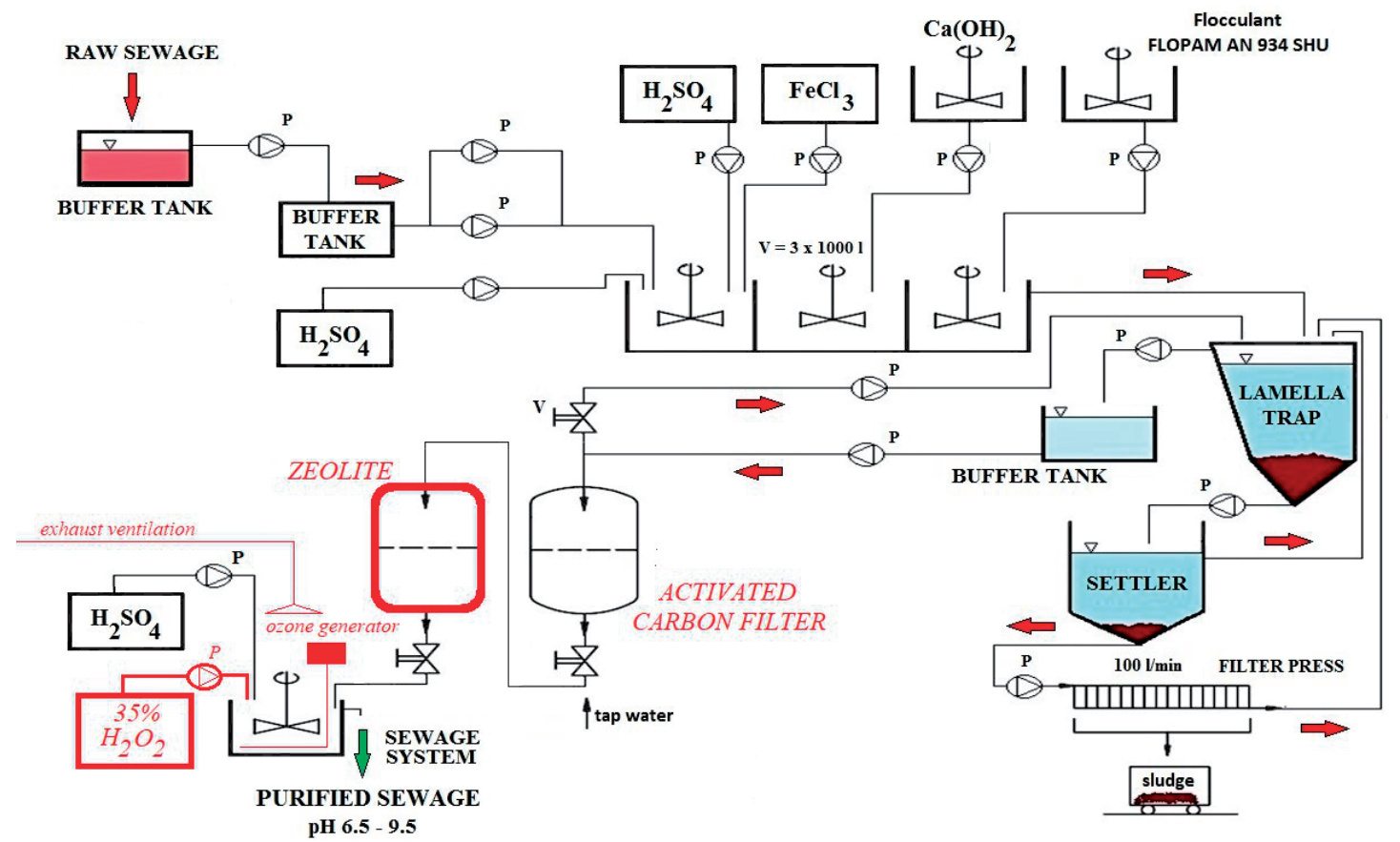

Fig. 9. A proposal for a new manganese phosphate coating wastewater treatment system.

with the addition of solution of iron chloride in the amount of $0.1 \mathrm{ml}$ and a dose of $\mathrm{H}_{2} \mathrm{O}_{2}$ in the amount of 1 and $2 \mathrm{ml}$. Samples of $5 \mathrm{ml}$ were taken every 5 minutes. The results of measurements of wastewater treatment using the Fenton method are shown in Fig. 6.

Conducting the Fenton process allowed to reduce the content of nitrates( $\mathrm{V})$ in raw sewage from $13 \mathrm{mg} / \mathrm{l}$ to the value of $0.031 \mathrm{mg} / \mathrm{l}$ at the dose of $\mathrm{H}_{2} \mathrm{O}_{2}$ $2 \mathrm{ml} / 500 \mathrm{ml}$ of the sewage sample and to the value of $0.012 \mathrm{mg} / 1$ at the dose of $3 \mathrm{ml}$ within 30 minutes without addition iron compound. Out of all five tests, the most favorable involved the addition of 2-3 ml hydrogen peroxide without iron(III) compound (Fig. 6). The results of the efficiency of wastewater treatment with the Fenton reaction in the system are presented in Table 7.

Nitrates(V) contained in the wastewater originate from the process baths containing nitrates, which are a process accelerator in the case of manganese phosphate coating. One of such compounds is manganese(II) nitrate(V), potassium nitrate(V). Nitrate(V) and also nitrite(III) and ammonia compounds decomposition proceeds according to two proposed chemical reactions:

$$
\begin{gathered}
2 \mathrm{KNO}_{3}+3 \mathrm{H}_{2} \mathrm{O}_{2}+\mathrm{H}_{2} \mathrm{SO}_{4} \rightarrow 4 \mathrm{H}_{2} \mathrm{O} \\
+\mathrm{K}_{2} \mathrm{SO}_{4}+3 \mathrm{O}_{2}+2 \mathrm{NO} \uparrow \\
2 \mathrm{KNO}_{2}+2 \mathrm{H}_{2} \mathrm{O}_{2}+2 \mathrm{H}_{2} \mathrm{SO}_{4} \rightarrow\left(\mathrm{NH}_{4}\right)_{2} \mathrm{SO}_{4} \\
+\mathrm{K}_{2} \mathrm{SO}_{4}+5 \mathrm{O}_{2} \\
2 \mathrm{NH}_{3}+3 \mathrm{H}_{2} \mathrm{O}_{2} \rightarrow 6 \mathrm{H}_{2} \mathrm{O}+\mathrm{N}_{2} \uparrow
\end{gathered}
$$

During the Fenton process, the turbidity of the sewage/hydrogen peroxide mixture was also measured.
For this purpose, five samples of raw sewage were prepared with a volume of $500 \mathrm{ml}$ and a variable dose of 1-5 $\mathrm{ml} \mathrm{H}_{2} \mathrm{O}_{2}$. Depending on the dose of hydrogen peroxide, the turbidity of the solution increases in direct proportion to the amount of oxidizing agent used, due to precipitation of manganese and iron oxides and hydroxides. The results are shown in Fig. 7.

\section{Sorption on Activated Carbon and Zeolite}

On the basis of the obtained test results, it can be concluded that passing the sewage through the system with activated carbon-zeolite effectively reduces iron, manganese and nitrates(V) (Fig. 8). However, this method does not give favorable effects in the case of reduction of phosphates( $\mathrm{V})$.

When analyzing the obtained results, it should be indicated that in the case of wastewater from manganese phosphate coating, this technique may be useful in the treatment of technological washers and recycling of water in a closed cycle, which will reduce the cost of phosphating. It can also be helpful in cleaning sewage already treated by coagulation.

Based on the conducted sorption of wastewater with the use of activated carbon and zeolite (Fig. 8), it can be concluded that this process does not bring much results in the reduction of orthophosphates. It can be stated that the sorption capacity is low in relation to orthophosphates. The bed breakthrough took place after $200 \mathrm{ml}$ of raw sewage had been passed through. Sorption has been used to remove contaminants such as nitrates, iron and manganese. After passing 2,000 ml of raw sewage through a system containing $100 \mathrm{~g}$ of filling, the iron concentration was reduced from 
$2.70 \mathrm{mg} / 1$ to $1.25 \mathrm{mg} / 1$, the manganese concentration from $11.0 \mathrm{mg} / \mathrm{l}$ to $0.95 \mathrm{mg} / \mathrm{l}$, and the nitrate concentration from $10 \mathrm{mg} / \mathrm{l}$ up to $1.9 \mathrm{mg} / \mathrm{l}$ (Fig. 8).

Wastewater treatment in the activated carbonzeolite system allowed the reduction of nitrates( $\mathrm{V})$ by $81 \%$, iron by an average of $54 \%$ and manganese by an average of $91.4 \%$. This method is useful in cleaning washers and returning water in a closed system due to the rapid breakthrough of the bed in a setting of phosphate content. In the case of other parameters, the technical use of a bed with a mixed filling of activated carbonzeolite would reduce the amount of tap water used.

Based on the obtained results of research on raw sewage from manganese phosphate coating, a new sewage treatment system was proposed using several methods; it is demonstrated in Fig. 9. It proposes the installation of a pressure filter with a zeolite bed assembled just before the filter filled with activated carbon. Another new element is the installation of a tank with a hydrogen peroxide solution with an automatic dosing pump that would feed a certain amount of $\mathrm{H}_{2} \mathrm{O}_{2}$ during the discharge of sewage to the municipal sewage system. Such a solution will effectively reduce the removed nitrates $(\mathrm{V})$, thus decreasing the burden of nitrogen compounds in the municipal sewage treatment plant.

A new optional solution is also the installation of an ozone generator with a feeding nozzle, which would additionally oxidize the residual nitrates(V) removed in the sewage. A major element in the structure is the installation of an air extraction system which will remove the residual unreacted ozone; this would allow safe operation of the entire installation due to the toxicity of the generated ozone (Fig. 9).

\section{Conclusions}

The treatment of wastewater from manganese phosphate coating by coagulation using iron(III) chloride and waste sulfuric(VI) acid contaminated with iron(III) phosphate allowed to reduce the content of phosphates by $93.6 \%$, nitrate(V) by $53.6 \%$, iron by $79.5 \%$ and manganese by $91.1 \%$.

Wastewater treatment using sorption in the activated carbon-zeolite system allowed to reduce $91.4 \%$ manganese content, 54\% iron content on average and $81 \%$ nitrate(V) content. However, the method was not successful in reducing phosphate(V).

Based on the obtained results of measurements of sewage treatment with the Fenton method, the most advantageous proved to be the trial with the addition of 2-3 $\mathrm{ml}$ hydrogen peroxide without iron(III) compound due to the residual iron content in the tested sewage. The Fenton method also requires the use of mechanical filtration due to the elevated metabolic rate in wastewater.

For ozone treatment of sewage, this method reduced phosphates by $29.8 \%$ in the case of raw sewage and by
$40 \%$ in the case of treated sewage. The method also reduced the content of nitrates by $60 \%$ in the case of raw sewage and by $91.6 \%$ in the content of iron in the raw sewage, and on average by $98.3 \%$ for the sewage treated with the coagulation method. Ozonation of wastewater allowed to reduce manganese content by $77.7 \%$. However, due to the increase in the amount of suspended solids in the vicinity of the turbidity index, ozonation also requires filtration.

A new solution was brought forward in waste water treatment by installing an ozone generator with an exhaust air extraction system, assembly of a pressure filter with zeolite filling and a tank with a hydrogen peroxide solution just before the discharge of wastewater into the sewage system.

\section{Conflict of Interest}

The authors declare no conflict of interest.

\section{References}

1. United Nations World Water Assessment Programme. The United Nations World Water Development Report 2017, Wastewater: The Untapped Resource, UNESCO, Paris, 2017.

2. RAI M.K., SHAHI G., MEENA R., CHAKRABORTY S., SINGH R.S., RAI B.N. Removal of hexavalent chromium $\mathrm{Cr}(\mathrm{VI})$ using activated carbon prepared from mango kernel activated with $\mathrm{H}_{3} \mathrm{PO}_{4}$. Resource-Efficient Technologies, 2 (1), S63, 2016.

3. WOLS B.A., HOFMAN-CARIS C.H.M. Review of photochemical reaction contents of organic micropollutants required for UV advanced oxidation processes in water, Water Res. 46, 2815, 2012.

4. LOOSDRECHT M.C.M., NIELSEN P.H., LOPEZVAZQUEZ C.M.L., BRDJANOVIC D., Experimental methods in wastewater treatment, IWA Publishing, 1, 2016.

5. DUSZCZYK J. Analysis of the content of pollutants in surface water in the commune of Ostrzeszów, Intrastructure and Ecology of Rural Areas, 3 (1), 291, 2019.

6. SZWACZYNA K., NIEWIARA E. Wpływ procedury przygotowania próbki ścieków koksowniczych na chromatograficzne oznaczanie wybranych Wielopierścieniowych węglowodorów aromatycznych(WWA), Analit, 6, 20, 2018.

7. ZHANG Z., ZHENG H., SUN Y., ZHAO CH., ZHOU Y., TANG X., ZHAO CH., A combined process of chemical precipitation and flocculation for treating phosphating wastewater, Desalination and Water Treatment, $1,2016$.

8. CHANAKYA K., MAHINDRA K. Removal of heavy metals from industrial waste water using coconut coir, International Journal of Civil Engineering and Technology, 8 (4), 2017.

9. SAURABH M.J. PARAG R.G. Intensification of industrial wastewater treatment using hydrodynamic cavitation combined with advanced oxidation at operating capacity of 70 L, Ultrasonics Sonochemistry, 52, 375, 2019. 
10. SALGOT M., FOLCH M. Wastewater treatment and water reuse, Current Option in Environmental Science \& Health, 2, 64, 2018

11. KORUS I., Galvanic wastewater treatment by means of anionic polymer enhanced ultrafiltration, ECOL CHEM ENG S, 19 (1), 19, 2012

12. INCE M., INCE K.O. An overview of adsorption technique for heavy metal removal from water/wastewater: A critical review. Int. J. Pure Appl. Sci. 3 (2), 10, 2017.

13. SHON H., PHUTSHO S., CHAUDHARY D.S., VIGNESWARAN S., CHO J. Nanofiltration for water and wastewater treatment- a mini review. Drinking Water Engineering and Science, Copernicus GmbH (Copernicus Publications) on behalf of the Delft University of Technology (TU Delft), 2013.

14. KORENAK J. NIELSEN H.C., BUKSEK H., PETRINIĆ I. Efficiency and economic feasibility of forward osmosis in textile wastewater treatment. Journal of Cleaner Production, 210, 1483, 2019.

15. DONG Y., LIU J., SUI M., QU Y., AMBUCHI J., WANG H., FENG Y. A combined microbial desalination cell and electrodialysis system for copper-contaning wastewater treatment and high-salinity-water desalination. Journal of Hazardous Materials, 321, 307, 2017.

16. CAPRARESCU S., PURCAR V., VAIREANU D.I., Separation of copper ions from synthetical prepared electroplating wastewater at different operating conditions using electrodialysis. Separation Science and Technology, Taylor \& Francis, 47 (16), 2273, 2012.

17. AKHTER M., HABIB G., QAMAR S.U. Application of Electro dialysis in Waste Water Treatment and Impact of Fouling on Process Performance, Journal of Membrane, (2), 2018

18. HEMBACH N., ALEXANDER J., HILLER Ch., WIELAND A., SHWARTZ T. Dissemination prevention of antibiotic resistant and facultative phatogenic bacteria by ultrafiltration and ozone treatment at an urban wastewater treatment plant. Nature-scientific reports, 9, 12843, 2019.

19. JIAND M., YE K., DENG J., LIN J., YE J., ZHAO S., BRUGGEN B.V. Conventional ultrafiltration as effective strategy for dye fractionation in textile wastewater treatment. Environ. Sci. Technol. 52 (18), 2018.

20. ACHARYA J., SAHU J.N., SAHOO B.K., MOHANTY C.R., MEIKAP B.C. Removal of chromium(VI) from wastewater by activated carbon development from Tamarind wood activated with zinc chloride. Chemical Engineering Journal, Elsevier, 57 (5), 2006, 2014.

21. ADEMILUYI F.T., AMADI S.A., AMAKAMA N.J. Adsorption and Treatment of Organic Contaminations using Activated Carbon from Waste Nigerian Bamboo. Journal of Applied Sciences and Environmental Management, 4, 39, 2016.

22. STREIT A., COLLAZZO C.G, DRUZIAN S.P., VERDI R.S., FOLETTO E.L., OLIVIERA L.F.S., DOTTO G.L. Adsorption of ibuprofen, ketoprofen, and paracetamol onto activated carbon prepared from effluent treatment plant sludge of the beverage industry. Chemosphere, 128322, 2020.

23. DELARGO A.G.A, LUGO-LUGO V., HERNANDEZ I.L., MIRANDA V.M., FUENTES-RIVAS R.M., URENANUNEZ F., Industrial wastewater treated by galvanic, galvanic Fenton, and hydrogen peroxide system, Journal of Water Processing Engineering, 22, 1, 2018.

24. KHAN J., TARIQ M., MUHAMMAD M., MEHMOOD M.H., ULLAH I., RAZIQ A., AKBAR F., SAQIB M.,
RAHIM A., NAIZ A. Kinetic and thermodynamic study of oxidative degradation of acid yellow 17 dye by Fentonlike process: Effect of $\mathrm{HCO}_{3}{ }^{-}, \mathrm{CO}_{3}{ }^{2-}, \mathrm{Cl}^{-}$and $\mathrm{SO}_{4}{ }^{2-}-$ on dye degradation, 33 (2), 2019.

25. BARTKIEWICZ B., UMIEJEWSKA K., Oczyszczanie ścieków przemysłowych, PWN, 2010.

26. ZARZYCKI R., IMBIEROWICZ M., STELMACHOWSKI M., Wprowadzenie do inżynierii i ochrony środowiska, WNT, 2007.

27. BHARGAVA A. Physico-Chemical Waste Water Treatment Technologies: An Overview, 2016.

28. THE CH.Y., BUDIMAN P.M., PUI YE SH. K., WU Y. Recent advancement of coagulation-flocculation and its application in wastewater treatment. Ind. Eng. Chem. Res., 55 (16), 4363, 2016.

29. DA ROSA J.J., RUBIO J. The FF (flocculation-flotation) process. Minerals Engineering, 18 (7), 701, 2005.

30. RITESH P., SUBRATA KUMAR M., ANUGRAH S., Flotation technique: Its mechanisms and design parameters, Chemical Engineering and Processing Process Intensification, 127, 249, 2018.

31. DELIYANNI E.A., KYZAS G.Z., MATIS K.A. Various flotation techniques for metal ions removal, Journal of Molecular Liquids, 225, 260, 2017.

32. RUBIO J., SOUZA M.L., SMITH R.W. Overview of flotation as a wastewater treatment technique. Minerals Engineering, Elsevier, 15 (3), 139, 2002.

33. DUSZCZYK J., SIUZDAK K., KLIMCZUK T., STRYCHALSKA-NOWAK J., ZALESKA-MEDYNSKA, A. Manganese Phosphatizing Coatings: The Effects of Preparation Conditions on Surface Properties. Materials, 11, 2585, 2018.

34. DUSZCZYK J., Zagrożenia w pracy laboranta galwanicznego; Bezpieczeństwo Pracy: nauka i praktyka, 1, 11-14, 2019 [In Polish].

35. BARBUSIŃSKI K., MAJEWSKI J. Discoloration of azo dye acid red 18by Fenton reagent in the presence of iron powder. Pol. J. EnvironStud., 12, 151-15, 2003.

36. CARNEIRO P.A., NOGUEIRA R.F.P., ZANONI M.V.B. Homogeneous photodegradation of C, I, Reactive Blue 4 using a photo-Fenton process under artificial and solar irradiation. Dyes Pigments, 74, 127, 2007.

37. BALI U., KARAGÖZOĞLU B., Performance comparison of Fenton process ferric coagulation and $\mathrm{H}_{2} \mathrm{O}_{2}$ /pyridine/ $\mathrm{Cu}$ (II) system fordecolorization of remazol turquoise blue G133. Dyes Pigments, 74, 73, 2007.

38. KABITA D., SUBRATA M., SEKHAR B., BASAB CH. Chemical oxidation of methylene blue using a Fenton-like reaction, Journal of Hazardous Materials, B84, 57, 2001.

39. PERVEZ M.D., FELIX TELEGIN Y., CAIL Y., XIA D., ZARRA T., NADDEO V. Efficient Degradation of Mordant Blue 9 Using theFenton-Activated Persulfate System, Water, 11, 2532, 2019.

40. ASHRAF S.S., RAUF M.A., SEHAM A., Degradation of Methyl Red using Fenton's reagentand the effect of various salts, Dyes and Pigments, 69, 74, 2006.

41. Polish Standard, PN-EN ISO 5667-10 Jakość Wody. Pobieranie próbek. Wytyczne pobierania próbek ścieków, 2017.

42. Ordinance of the Minister of Infrastructure and Development of August 25, 2015, amending the ordinance on the performance of the obligations of industrial wastewater suppliers and the conditions for discharging wastewater into sewage systems, Journal of 2015, item 1456. 